\title{
The 1.5 Ms observing campaign on IRAS 13224-3809 - I. X-ray spectral analysis
}

\author{
J. Jiang(姜嘉陈.) ${ }^{1}{ }^{\star}$ M. L. Parker, ${ }^{1,2^{\star}}$ A. C. Fabian, ${ }^{1}$ W. N. Alston, ${ }^{1}$ D. J. K. Buisson, ${ }^{1}$ \\ E. M. Cackett, ${ }^{3}$ C.-Y. Chiang, ${ }^{3}$ T. Dauser, ${ }^{4}$ L. C. Gallo,${ }^{5}$ J. A. García, ${ }^{4,6}$ \\ F. A. Harrison, ${ }^{6}$ A. M. Lohfink, ${ }^{7}$ B. De Marco, ${ }^{8}$ E. Kara, ${ }^{9}$ J. M. Miller, ${ }^{10}$ \\ G. Miniutti, ${ }^{11}$ C. Pinto, ${ }^{1}$ D. J. Walton ${ }^{1}$ and D. R. Wilkins ${ }^{12}$ \\ ${ }^{1}$ Institute of Astronomy, University of Cambridge, Madingley Road, CB3 OHA Cambridge, UK \\ ${ }^{2}$ European Space Agency (ESA), European Space Astronomy Centre (ESAC), E-28691 Villanueva de la Cañada, Madrid, Spain \\ ${ }^{3}$ Department of Physics and Astronomy, Wayne State University, 666 W, Hancock Street, Detroit, MI 48201, USA \\ ${ }^{4}$ Dr Karl Remeis-Observatory and Erlangen Centre for Astroparticle Physics, Sternwartstr. 7, D-96049 Bamberg, Germany \\ ${ }^{5}$ Department of Astronomy and Physics, Saint Mary's University, 923 Robie Street, Halifax, NS, B3H 3C3, Canada \\ ${ }^{6}$ Cahill Center for Astronomy and Astrophysics, California Institute of Technology, Pasadena, CA 91125, USA \\ ${ }^{7}$ Department of Physics, Montana State University, Bozeman, MT 59717-3840, USA \\ ${ }^{8}$ Nicolaus Copernicus Astronomical Center, Polish Academy of Sciences, Bartycka 18, PL-00-716 Warsaw, Poland \\ ${ }^{9}$ Department of Astronomy, University of Maryland, College Park, MD 20742-2421, USA \\ ${ }^{10}$ Department of Astronomy, University of Michigan, 1085 South University, Ann Arbor, MI 48109, USA \\ ${ }^{11}$ Centro de Astrobiología (INTA-CSIC), Dep. de Astrofísica, ESAC campus, E-28692 Villanueva de la Cañada, Spain \\ ${ }^{12}$ Kavli Institute of Particle Astrophysics and Cosmology, Standford University, 452 Lomita Mall, Stanford, CA 94305, USA
}

Accepted 2018 March 28. Received 2018 March 1; in original form 2017 September 11

\begin{abstract}
We present a detailed spectral analysis of the recent $1.5 \mathrm{Ms} X M M-N e w t o n$ observing campaign on the narrow-line Seyfert 1 galaxy IRAS 13224-3809, taken simultaneously with $500 \mathrm{ks}$ of NuSTAR data. The X-ray light curve shows three flux peaks, registering at about 100 times the minimum flux seen during the campaign, and rapid variability with a time-scale of kiloseconds. The spectra are well fit with a primary power-law continuum, two relativisticblurred reflection components from the inner accretion disc with very high iron abundance, and a simple blackbody-shaped model for the remaining soft excess. The spectral variability is dominated by the power-law continuum from a corona region within a few gravitational radii from the black hole. Additionally, blueshifted $\mathrm{Ne}$ x, Mg XII, Si XIv, and S XVI absorption lines are identified in the stacked low-flux spectrum, confirming the presence of a highly ionized outflow with velocity up to $v=0.267$ and $0.225 \mathrm{c}$. We fit the absorption features with xstar models and find a relatively constant velocity outflow through the whole observation. Finally, we replace the bbody and supersolar abundance reflection models by fitting the soft excess successfully with the extended reflection model relxil1D, which allows for higher densities than the standard relxill model. This returns a disc electron density $n_{\mathrm{e}}>10^{18.7} \mathrm{~cm}^{-3}$ and lowers the iron abundance from $Z_{\mathrm{Fe}}=24_{-4}^{+3} \mathrm{Z}_{\odot}$ with $n_{\mathrm{e}} \equiv 10^{15} \mathrm{~cm}^{-3}$ to $Z_{\mathrm{Fe}}=6.6_{-2.1}^{+0.8} \mathrm{Z}_{\odot}$.
\end{abstract}

Key words: accretion, accretion discs-black hole physics-galaxies: Seyfert-X-rays: galaxies.

\section{INTRODUCTION}

\subsection{Accretion disc-corona for AGN}

The primary X-ray emission from black holes can be described by a power-law continuum with a high-energy cut-off, often explained by inverse Comptonization of the thermal disc photons in a coronal region (e.g. Haardt \& Maraschi 1993). The corona is known to be compact, though its exact nature still remains unknown. Advanced imaging and timing analyses show the $\mathrm{X}$-ray emitting regions are highly compact and only a few gravitational radii from the black hole (Reis \& Miller 2013, for review). For example, the discovery of a soft X-ray reverberation lag of $30 \mathrm{~s}$ in $1 \mathrm{H} 0707-495$, a narrow line Seyfert 1 galaxy (NLS1), has indicated the X-ray emitting region is very compact (Fabian et al. 2009), which will be discussed more 
later. Moreover, in the microlensed galaxy the microlensing duration in the X-ray band is much shorter than the UV/optical band, which indicates a much smaller X-ray emitting region than the optical emitting region (e.g. Morgan et al. 2008; Chartas et al. 2017). Some progress on modelling the disc emissivity profile with different compact corona geometries has also been made (e.g. Wilkins et al. 2015; Wilkins \& Gallo 2015), and is consistent with a compact coronal region, close to the event horizon. The compact corona also agrees with the predictions of the Comptonization model with magnetic reconnection (e.g. Merloni \& Fabian 2001), where the corona is described as a region of smooth magnetic field with increasing strengths towards small radii. A coronal geometry in which the continuum originates from a small region on the spin axis at height $h$ above the central black hole can describe X-ray data from many luminous accreting black holes well (e.g. MCG-6-30-15 Fabian \& Vaughan 2003; Miniutti et al. 2003; Vaughan \& Fabian 2004). Such a geometry is usually called the 'lamp-post' geometry.

The reprocessing of the coronal radiation by the colder material in the disc produces a hump above $20 \mathrm{keV}$ and a series of atomic lines, most notably the strong $\mathrm{Fe} \mathrm{K} \alpha$ emission line at $6.4 \mathrm{keV}$. These features are referred to as the disc reflection component. The interaction between the primary power-law photons and the disc material can produce both emission, including fluorescence lines and recombination continuum, and absorption edges (George \& Fabian 1991; Ross \& Fabian 2005; García \& Kallman 2010). X-ray reflection off the inner part of the accretion disc is highly affected by strong gravitational effects including gravitational redshift and Doppler effects (e.g. Fabian et al. 1989; Reynolds \& Nowak 2003), which can offer information on the geometry of the corona and the spin of the central black hole. For example, relativistic broad Fe line features have been detected in the reflection spectra of many AGN sources, such as MCG-6-30-15 (e.g. Tanaka et al. 1995; Wilms et al. 2001; Fabian \& Vaughan 2003; Marinucci et al. 2014b), 1H0707495 (e.g. Fabian et al. 2004, 2009), NGC 1365 (e.g. Risaliti et al. 2013; Walton et al. 2014), Mrk 335 (e.g. Larsson et al. 2008; Gallo et al. 2013; Walton et al. 2013; Parker et al. 2014), IRAS 005217054 (e.g. Tan et al. 2012), NGC 3783 (e.g. Brenneman et al. 2011), and Swift J2127.4+5654 (e.g. Miniutti et al. 2009; Marinucci et al. 2014a). Recent work on AGN X-ray variability has shown time lags where the soft excess and $\mathrm{Fe} \mathrm{K} \alpha$ line lag behind the power-law continuum. Evidence of lags of $<100 \mathrm{~s}$, referred to as reverberation lags, has been detected in AGNs (e.g. Fabian et al. 2009; Zoghbi et al. 2010; Zoghbi, Uttley \& Fabian 2011; Zoghbi et al. 2012; De Marco et al. 2013; Kara et al. 2013a, 2016). Lags of a few milliseconds have also been detected in X-ray binaries (e.g. De Marco et al. 2015; De Marco \& Ponti 2016; De Marco et al. 2017), indicating similar processes are driving the spectral variability in both classes of black hole.

\subsection{IRAS 13224-3809}

IRAS $13224-3809(z=0.066$; Allen et al. 1991) is classified as a narrow line Seyfert-1 galaxy hosting a supermassive black hole ( $M=10^{6}-10^{7} \mathrm{M}_{\odot}$, Zhou \& Wang 2005). It has been studied in multiple bands previously. It was identified by Boller et al. (1997) as a radio quiet source, with no clear jet emission yet observed (1.4 GHz flux of $5.4 \mathrm{mJy}$, Feain et al. 2009). UV continuum variability of 24 per cent in $3 \mathrm{yr}$ has been observed (Rodriguez-Pascual, Mas-Hesse \& Santos-Lleo 1997). Leighly (2001) found asymmetric high ionization emission lines in the HST UV spectrum, indicating evidence of an outflow. $L y_{\alpha}$ line variability has been detected with variation in the line profile and flux level (Mas-Hesse et al. 1994).
Though there is no significant rapid optical variability detected (Young et al. 1999), it exhibits extreme and rapid variability in the $\mathrm{X}$-ray band on very short time-scales of hundreds of seconds (Boller et al. 1997; Gallo et al. 2004; Fabian et al. 2013)

IRAS 13224-3809 was observed by the XMM-Newton satellite (Jansen et al. 2001) in 2002 and 2011, showing a steep spectrum (e.g. $\Gamma=2.5-2.7$, Boller et al. 2003; Fabian et al. 2013) with an obvious soft excess below $1.5 \mathrm{keV}$ and a sharp spectral drop around $8 \mathrm{keV}$ (Boller et al. 2003; Ponti et al. 2010; Fabian et al. 2013; Chiang et al. 2015). The spectrum shows very strong Fe K and Fe L emission lines (Ponti et al. 2010; Fabian et al. 2013) that are explained as the indication of reflection off the inner accretion disc around a rapidly rotating black hole (e.g. $a_{*}=0.988 \pm 0.001$; Fabian et al. 2004, 2013). By analysing the disc emissivity profile in the $500 \mathrm{ks}$ long XMM-Newton observation in 2011, Fabian et al. (2013) found that the corona is located within a few gravitational radii. Chiang et al. (2015) fitted the soft excess in the EPIC spectra successfully with the combination of the reflection model reflionx (Ross \& Fabian 2005) and a blackbody. The RGS spectra do not exhibit any evidence for absorption features produced by a warm absorber (Pinto et al. 2017) or partial covering clouds (Chiang et al. 2015), and there is no evidence for absorption in the UV spectra (Leighly \& Moore 2004).

A soft $(0.3-1 \mathrm{keV})$ lag behind the $1-4 \mathrm{keV}$ band was found by Ponti et al. (2010), similar to the lag detected in 1H0707-495 (Fabian et al. 2009; Zoghbi et al. 2010), though the significance of the lags is limited due to the short duration of the XMM-Newton observation in 2002. A more detailed timing study of the longer observation in 2011 found a strong Fe $\mathrm{K}$ feature in the lag-energy spectrum and suggested that the frequency and the amplitude of the lag varies in high flux intervals and quiescent periods (Kara et al. 2013b). These changes in the lag properties with flux are thought to be due to a more compact disc/corona system. Chainakun, Young \& Kara (2016) simulated the time-averaged model and lagenergy model in the lamp-post scenario and the model also supports a reflection origin for the soft excess of IRAS 13224-3809 and an X-ray source very close to the central black hole $\left(h \approx 2 \mathrm{R}_{\mathrm{g}}\right.$ ).

IRAS 13224-3809 was recently observed with a XMM-Newton quasi-simultaneous 1.5 Ms and NuSTAR (Harrison et al. 2013) observing campaign in 2016 (P.I.: A. C. Fabian). In Parker et al. (2017b), we reported the discovery of a series of strongly blueshifted $(v=0.236 \pm 0.006 \mathrm{c})$ absorption lines from $\mathrm{O}, \mathrm{Ne}$, and $\mathrm{Fe}$ in the $X M M-N e w t o n$ EPIC-pn and RGS spectra, indicating the presence of an ultra-fast outflow (UFO). These features in IRAS 13224-3809 were found to be strongly dependent on the source flux, varying on time-scales as short as a few kiloseconds. Similar outflows have been detected in many sources by studying the blueshifted Fe Kshell absorption lines in the $7-10 \mathrm{keV}$ energy band (e.g. Tombesi et al. 2010), but none has ever been seen to vary as rapidly, or to have strong correlation with flux (Parker et al. 2017a; Pinto et al. 2017). The flux dependence can be explained as the increased source flux ionizing the outflowing gas, saturating the absorption lines. Alternatively, the absorption features could be produced in a layer on top of the accretion disc, where these relativistic velocities occur naturally. In this case, the change in strength of the absorption is due to the change in reflection fraction with flux, as only the reflected emission is absorbed (Gallo \& Fabian 2011). This model has been successfully applied to PG 1211+143 (Gallo $\&$ Fabian 2013). Following on from the detection of the UFO, in Parker et al. (2017a) we showed that the Fe xxv/Xxvi absorption features are strongly present in simple variability spectra, along with 
Table 1. XMM-Newton observation log. The duration is the length of scheduled observation. The 'annulus' column indicates where an annulus-shaped source region with an inner radius of 5 arcsec is used to extract spectra during the data reduction to reduce the pile-up effects. The usable percentage of each EPIC-pn exposure after correcting for flaring particle background is shown after the corresponding net exposure length. The observed flux is calculated by the best-fitting model for EPIC pn spectra in the $0.3-10 \mathrm{keV}$ band in the unit $10^{-12} \mathrm{erg} \mathrm{cm}^{-1} \mathrm{~s}^{-1}$.

\begin{tabular}{|c|c|c|c|c|c|}
\hline Revolution & Obs. ID & Start date & $\begin{array}{l}\text { EPIC pn net exposure }(\mathrm{ks}) \\
\text { and usage percentage }\end{array}$ & $\begin{array}{c}\text { Observed flux } \\
\left(10^{-12} \mathrm{erg} \mathrm{cm}^{-1} \mathrm{~s}^{-1}\right)\end{array}$ & Annulus \\
\hline 3037 & 0780560101 & 2016-07-08, 19:33:33 & 18 (95 per cent), 36 (92 per cent) & $3.68 \pm 0.01$ & MOS and pn \\
\hline 3038 & 0780561301 & 2016-07-10, 19:25:31 & $122(94$ per cent $)$ & $3.77 \pm 0.01$ & MOS and pn \\
\hline 3039 & 0780561401 & 2016-07-12, 19:34:13 & 78 (94 per cent), 36 (95 per cent) & $3.27 \pm 0.01$ & - \\
\hline 3043 & 0780561501 & $2016-07-20,19: 01: 53$ & $120(98$ per cent $)$ & $2.57 \pm 0.01$ & - \\
\hline 3044 & 0780561601 & $2016-07-22,18: 36: 58$ & 118 (98 per cent) & $5.66 \pm 0.01$ & MOS and pn \\
\hline 3045 & 0780561701 & $2016-07-24,18: 28: 28$ & 117 (97 per cent) & $3.15 \pm 0.01$ & MOS \\
\hline 3046 & 0792180101 & 2016-07-26, 18:18:44 & 123 (98 per cent) & $3.36 \pm 0.01$ & MOS and pn \\
\hline 3048 & 0792180201 & 2016-07-30, 18:02:21 & 120 (98 per cent) & $3.52 \pm 0.01$ & MOS and pn \\
\hline 3049 & 0792180301 & 2016-08-01, 17:54:51 & 108 (99 per cent) & $1.53 \pm 0.01$ & - \\
\hline 3050 & 0792180401 & $2016-08-03,17: 47: 25$ & 108 (94 per cent) & $8.05 \pm 0.01$ & MOS and pn \\
\hline 3052 & 0792180501 & 2016-08-07, 17:40:58 & 112 (97 per cent) & $3.21 \pm 0.01$ & MOS \\
\hline 3053 & 0792180601 & 2016-08-09, 18:29:52 & 116 (97 per cent) & $7.84 \pm 0.01$ & MOS and pn \\
\hline
\end{tabular}

the Mg XII, Si XIV, S XVI, Ar XVIII, and Ca Xx Ly $\alpha$ lines. Again, all of these features are strongly flux dependent and extremely rapidly variable.

IRAS $13224-3809$ is well known as a twin to another bright, rapidly variable NLS1: $1 \mathrm{H}$ 0707-495, which also shows very strong $\mathrm{Fe} L$ and $K$ emissions in the XMM-Newton spectrum (Fabian et al. 2009). A reverberation lag of $30 \mathrm{~s}$ from the bright $\mathrm{Fe} L$ emission line was found by Fabian et al. (2009) and Zoghbi et al. (2010). Kara et al. (2013a) found a low-frequency hard lag corresponding to the disc fluctuation propagation, and a high-frequency soft lag corresponding to the time delay between the coronal emission and the disc reflection in a $1.3 \mathrm{Ms}$ observing campaign. In addition, Dauser et al. (2012) first found a highly ionized outflowing wind with changing velocity from 0.11 to $0.18 \mathrm{c}$ by studying the $500 \mathrm{ks}$ long XMM-Newton and $120 \mathrm{ks}$ Chandra quasi-simultaneous observations. Boller et al. (2002) found a sharp spectral drop around $7 \mathrm{keV}$ in the $X M M-$ Newton spectra, suggesting a neutral Fe $K$ edge. Hagino et al. (2016) interpreted the absorption feature above $7 \mathrm{keV}$ with a disc wind model. More data analysis on the ionized outflow in this source will be published in near future.

In this work, we analyse the $1.5 \mathrm{Ms} X M M-N$ ewton observing campaign of IRAS 13224-3809 using broad-band spectroscopy. We focus on the spectral characteristics of the AGN, and compare our results with previous studies. In Section 2, we briefly describe the data reduction and briefly introduce the X-ray variability shown in the light curve; in Section 3, we focus on the stacked spectral analysis; in Section 4, we study the spectral differences between different flux levels; in Section 5, we divide the whole exposure into 12 slices and study the spectral variability. A detailed timing analysis, including further reverberation lag studies will be presented in future work (Alston et al., in preparation) and the RGS spectral analysis is in an independent paper (Pinto et al. 2018).

\section{DATA REDUCTION AND LIGHT CURVES}

Our data analysis is conducted using all the data from the 2016 $X M M-$ Newton observing campaign. A list of the observations used in this paper for spectral analysis, with a total exposure of $\approx 1.5 \mathrm{Ms}$, is given in Table 1. A list of quasi-simultaneous NuSTAR observations used in this work is shown in Table 2.

\subsection{XMM-Newton Data Reduction}

We reduce the XMM-Newton data using V15.0.0 of the $X M M-$ Newton Science Analysis System (SAS) software package and calibration files (ccf) v.20160201. First, the clean calibrated event lists are created by running EMPROC (for EPIC-MOS data) and EPPROC (for EPIC-pn data). Good time intervals are selected by removing intervals dominated by flaring particle background, defined as intervals where the single event (PATTERN $=0$ ) count rate in the $>10 \mathrm{keV}$ band is larger than 0.35 counts $\mathrm{s}^{-1}$ for EPIC-MOS data and that in the $10-12 \mathrm{keV}$ band larger than 0.4 counts $\mathrm{s}^{-1}$ for EPIC-pn data. By running the EVSELECT task, we select single and double events for EPIC-MOS (PATTERN $<=12$ ) and EPICpn $($ PATTERN $<=4$, FLAG $=0)$ source event lists from a circular source region with radius of 35 arcsec. An annulus shaped source region with an inner radius of 5 arcsec is chosen to reduce pile-up if necessary for the observations with a very high count rate (see the last column of Table 1). The inner radius is chosen by running epatplot, with which the observed/model singles and doubles pattern fractions ratios are consistent with 1 within statistical errors. The EPIC-MOS cameras were being operated in the Small Window (SW) mode while the EPIC-pn camera was being operated in the Large Window (LW) mode. Background regions are chosen as circular regions with radii of 40 arcsec, close to the source region in the same unit on the camera. The background count rate remains $\approx 0.1 \mathrm{cts} \mathrm{s}^{-1}$. Then we create redistribution matrix files and auxiliary response files by running RMFGEN and ARFGEN tasks separately. We consider the EPIC spectra between 0.3 and $10 \mathrm{keV}$, unless otherwise specified. ADDSPEC is used to make a stacked spectrum for each camera, along with corresponding background spectra and response matrix files. The averaged EPIC-pn background count rate is $0.05205 \pm 0.00001 \mathrm{cts} \mathrm{s}^{-1}$ and the averaged EPIC-pn source count rate is $1.649 \pm 0.001 \mathrm{cts} \mathrm{s}^{-1}$.

\subsection{NuSTAR data reduction}

IRAS 13224-3809 was observed by the NUSTAR satellite seven times, listed in Table 2. The first observation interval (Obs IDs 60202001002, 60202001004, and 60202001006) was performed continuously followed by the second (Obs IDs 60202001008 and 602002001010) and the third intervals (Obs IDs 60202001012 and 
Table 2. NuSTAR observation log. Similar with Table 1. The observed flux is the total flux of the best-fitting model for FPMA and FPMB spectra in the $3-30 \mathrm{keV}$ band in the unit of $10^{-13} \mathrm{erg} \mathrm{cm}^{-1} \mathrm{~s}^{-1}$.

\begin{tabular}{lccc}
\hline Obs. ID & Start date & $\begin{array}{c}\text { Duration } \\
(\mathrm{ks})\end{array}$ & $\begin{array}{c}\text { Observed flux } \\
\left(10^{-13} \mathrm{erg} \mathrm{cm}^{-1} \mathrm{~s}^{-1}\right)\end{array}$ \\
\hline 60202001002 & $2016-07-08,19: 36: 08$ & 67.6 & $5.2 \pm 0.1$ \\
60202001004 & $2016-07-10,20: 01: 08$ & 67.4 & $6.0 \pm 0.3$ \\
60202001006 & $2016-07-12,18: 36: 08$ & 67.5 & $6.2 \pm 0.3$ \\
60202001008 & $2016-07-23,19: 01: 08$ & 70.2 & $8.2 \pm 0.4$ \\
60202001010 & $2016-07-27,02: 16: 08$ & 62.6 & $5.0 \pm 0.3$ \\
60202001012 & $2016-08-01,07: 46: 08$ & 171.7 & $7.8 \pm 0.2$ \\
60202001014 & $2016-08-08,05: 46: 08$ & 136.9 & $9.8 \pm 0.3$ \\
\hline
\end{tabular}

60202001014), each slightly separated in order to better overlap with the XMM-Newton observations. We reduce the NuSTAR data using the standard pipeline NUPIPELINE V0.4.5, part of HEASOFT V6.19 package, and instrumental responses from NuSTAR caldb V20161021. Source spectra are selected from circular regions with radii of 35 arcsec, and the background is obtained from nearby circular regions with radii of 120 arcsec. Spectra are extracted from the cleaned event files using NUPRODUCTS for both FPMA and FPMB. The observed flux in the $3-30 \mathrm{keV}$ band is also added in Table 2.

\subsection{Light curves and time variability}

The $0.3-10 \mathrm{keV}$ EPIC pn light curve of 2016 observation is shown in the middle panel of Fig. 1. The data are grouped into bins with interval widths of $3 \mathrm{ks}$. Extreme flux peaks $\left(12 \mathrm{ct} \mathrm{s}^{-1}\right)$ happened three times during the $1.5 \mathrm{Ms}$ observing campaign with the count rate three times the average level $\left(4 \mathrm{ct} \mathrm{s}^{-1}\right)$ and 100 times the lowest level $\left(0.13 \mathrm{ct} \mathrm{s}^{-1}\right)$. The $0.3-10 \mathrm{keV}$ band light curve shows rapid variability with a time-scale of kiloseconds. The first flux peak happened in the gap between two NUSTAR observations, but the NuSTAR FPMs capture the second and the third flux peak in the $X M M-N e w t o n$ observation. This new observing campaign captures stronger flux peaks, compared with the light curves of the $X M M-$ Newton observations in 2011 (e.g. $8 \mathrm{ct} \mathrm{s}^{-1}$ in Chiang et al. 2015).

Fig. 2 shows the unfolded spectra of all the XMM-Newton and NUSTAR observations against a constant model. The $1-4 \mathrm{keV}$ band shows 10 times flux difference between the highest (Obs ID 0780561601) and the lowest flux state (Obs ID 0792180301), while the iron band (4-7 keV) and the hard band (>10keV) show approximately three times flux difference and smaller variability than the $1-4 \mathrm{keV}$ band. The averaged spectrum shows a strong soft component below $1 \mathrm{keV}$ and a very broad strong $\mathrm{Fe} \mathrm{K} \alpha$ emission line at the 4-7 keV band. The spectrum becomes softer and shows a weaker $\mathrm{Fe} \mathrm{K} \alpha$ emission at higher flux states.

In order to group the spectra and reconstruct the response matrix in a more optimal way, we use the method in Kaastra \& Bleeker (2016) by taking both every bin's averaged energy and photon counts into account. ${ }^{1}$ The unfolded spectra against a constant model of all the observations show shown in Fig. 2. The flux at the iron band and the $<1 \mathrm{keV}$ band is less variable than that in the $1-4 \mathrm{keV}$ band (approximately 10 times difference).

For the spectral analysis, we use the XSPEC (12.9.1k) software package (Arnaud 1996) to fit all the spectra discussed, and Cstatistics (Cash 1979) is considered in this work, as required by

\footnotetext{
1 The PYTHONcode is written by Carlo Ferrigino. https://cms.unige.ch/ isdc/ferrigno/developed-code/.
}

the spectral binning method. The $\chi^{2}$ test is not used due to possible biased estimation of errors (Kaastra \& Bleeker 2016). The Galactic column density towards IRAS $13224-3809$ is fixed at the nominal value $5.3 \times 10^{20} \mathrm{~cm}^{-2}$ from Kalberla et al. (2005) if not specified. The column density calculated by the method in Willingale et al. (2013) is $6.78 \times 10^{20} \mathrm{~cm}^{-2}$ after accounting for the effect of molecular hydrogen. In the end of our stacked spectral analysis in Section 3.3, we will measure the Galactic column density with our X-ray spectra and fix it at our best-fitting value in the following analysis. The photoionization cross-section is from Balucinska-Church \& McCammon (1992) and He cross-section is from Yan, Sadeghpour \& Dalgarno (2001). The solar abundances of Wilms, Allen \& McCray (2000) were used. The fit parameters are reported in the observed frame. The quoted errors of best-fitting parameter values are at the 90 per cent confidence level. The bestfitting model parameters are all reported in the observer's frame unless explained explicitly. We assume the cosmological parameters $H_{0}=73 \mathrm{~km} \mathrm{~s}^{-1} \mathrm{Mpc}^{-1}, \Omega_{\text {matter }}=0.27$, and $\Omega_{\text {vacuum }}=0.73$. An isotropic model of emission is adopted when calculating the source luminosity. For local galactic absorption, the tbnew model (Wilms et al. 2000) is used. An additional constant model constant has been applied to vary normalizations between different instruments to account for calibration uncertainties.

Hereafter, black points with error bars are for FPMA; red points are for FPMB; green points are for MOS1; blue points are for MOS2; dark red points are for $\mathrm{pn}$ in figures unless explained specifically.

\section{TIME-AVERAGED SPECTRAL ANALYSIS}

In this section, we focus on studying the ionized reflection from the accretion disc around the central BH and identifying UFO absorption lines by analysing the time-averaged spectra. We first fit $X M M-N e w t o n$ and NUSTAR data independently and then a quasisimultaneous spectral analysis is conducted on both sets of data. The best-fitting model will be used as a template for further spectral analysis in Sections 4 and 5.

\subsection{Continuum fit}

\subsubsection{NuSTAR FPM data}

We first fit the stacked FPMA and FPMB spectra with an absorbed power law. The ratio plot in the top panel of Fig. 3 shows a broad Fe line feature present around $6.4 \mathrm{keV}$ and a Compton hump above $10 \mathrm{keV}$. To fit the reflection feature, an extended version of reflionx (Ross \& Fabian 2005) with wider iron abundance range (up to 30 times solar abundance) is used. The $\mathrm{Fe} K \alpha$ line in reflionx is treated as the recombination lines of Fe XXV and 


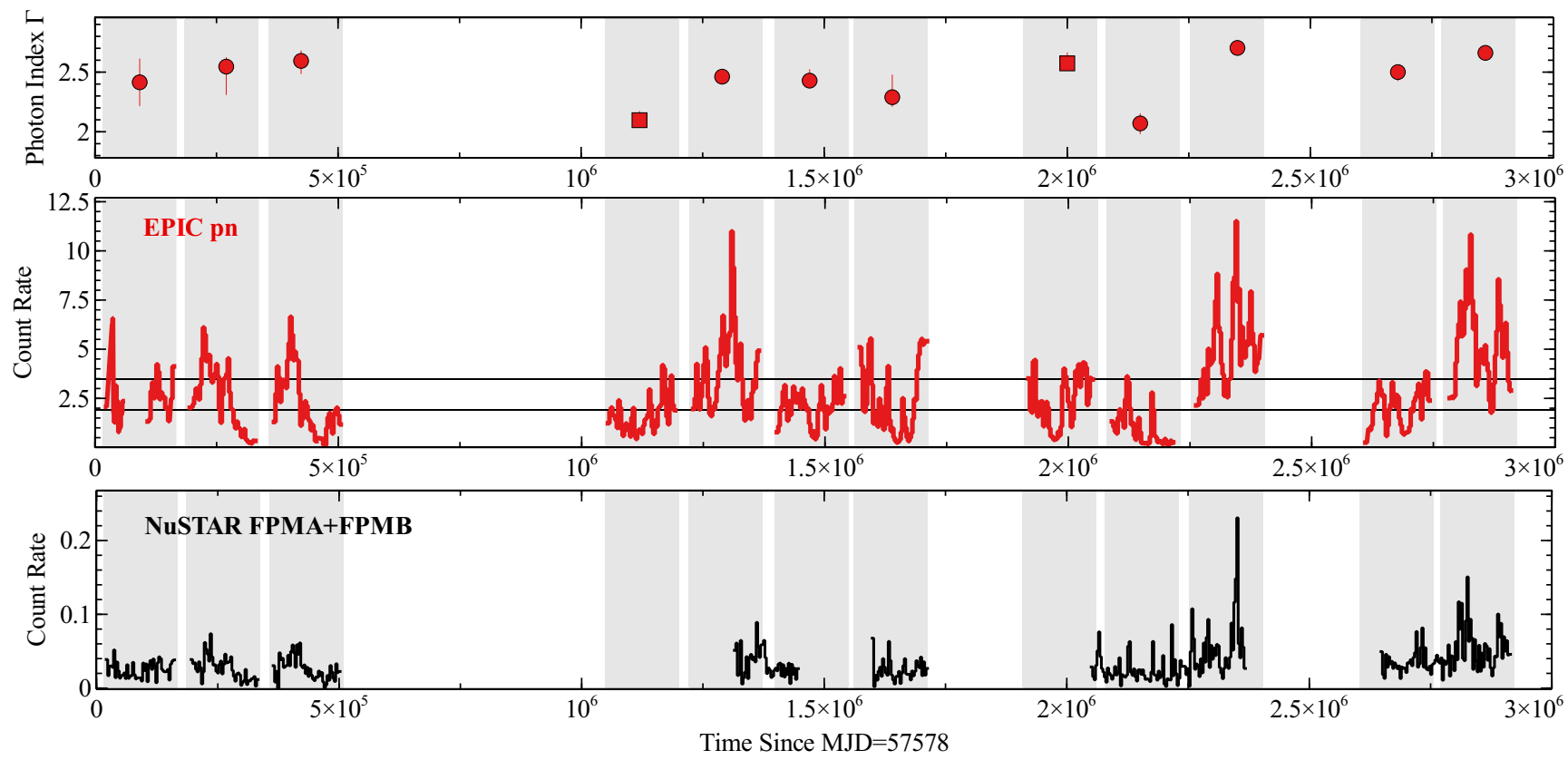

Figure 1. Top: the best-fitting photon index of the power-law continuum $\Gamma$ versus time. (See Section 5 for more details). The circles are the best-fitting values obtained by analysing the NUSTAR FPM and XMM-Newton EPIC spectra simultaneously, while the squares are the ones obtained by analysing only the $X M M-N e w t o n$ EPIC spectra. Middle: the $0.3-10 \mathrm{keV}$ EPIC pn light curve with $3 \mathrm{ks}$ bins. The observing campaign is divided into 12 observation intervals, each of around $120 \mathrm{ks}$. The time coverage is shown in the grey shaded region. A time-resolved spectral analysis on each observation is introduced in Section 5. The light curve is also divided into three flux intervals, each with a similar number of counts, by horizontal solid lines. A flux-resolved spectral analysis of the three flux states is introduced in Section 4. Three peaks $\left(12 \mathrm{cts} \mathrm{s}^{-1}\right)$ are detected during the whole observation. Bottom: the 3.0-78.0 keV NuSTAR FPMA+FPMB $3 \mathrm{ks}$ light curve. The time axis has been coordinated with the XMM-Newton EPIC pn light curve. The NuSTAR observing campaign is separated into seven intervals. The 4th, 6th, and 7th NUSTAR observations are divided into two time slices to conduct simultaneous time-resolved spectral analysis with the XMM-Newton data in Section 5.

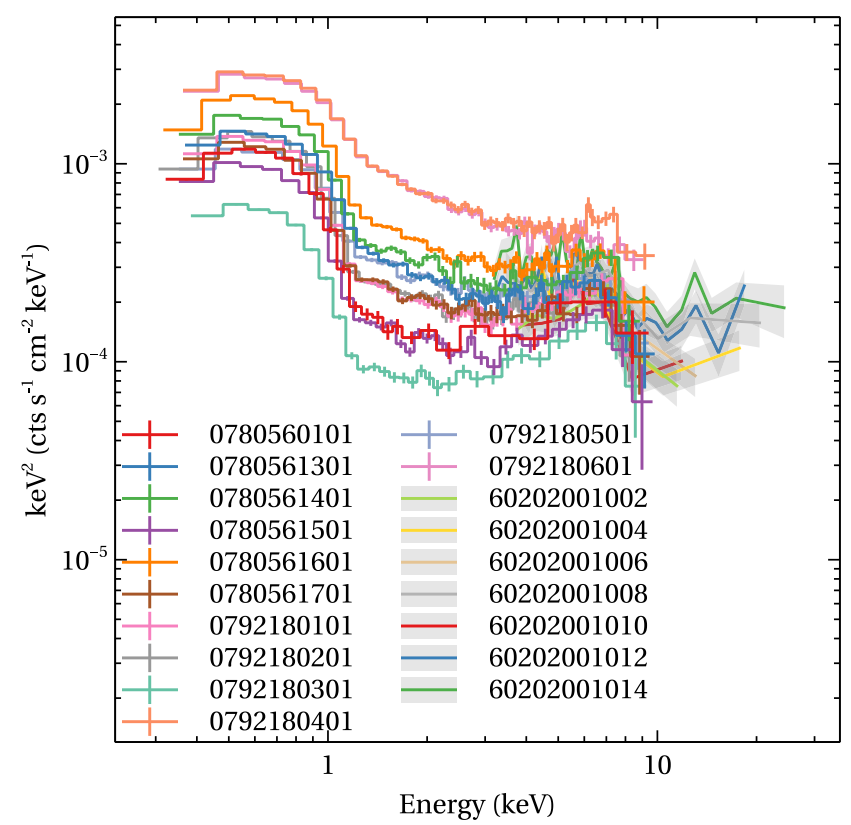

Figure 2. The unfolded spectra against a constant model of all the XMMNewton (lines with errorbars) and NUSTAR (shaded lines) observations. The spectra have been grouped for clarity.

Fe XXVI combined with the fluorescence lines of Fe VI-XVI. relconv is convolved with the local reflection model for relativistic effects (Dauser et al. 2013). After adding the relativistic reflection model relconv ${ }^{\star}$ reflionx, it reduces the statistics to
$C-s t a t / v=114.50 / 102^{2}$ (see the bottom panel of Fig. 3). The best-fitting model parameters are listed in Table 3. The fit requires a central black hole with spin $a_{*}>0.94$ viewed from an inclination angle of $i=54 \mathrm{deg}$. The fit puts a weak constraint on the iron abundance $\left(Z_{\mathrm{Fe}}<18\right)$. In order to test a possible low-energy cut-off in the broad-band spectrum, the high-energy cut-off parameter is allowed to vary and we obtained $E_{\text {cut }}>15 \mathrm{keV}(2 \sigma)$ by fitting the 3-30 keV band FPM spectra (refer to Fig. 4). Only the lower limit of the cut-off can be obtained. The curvature of the high-energy emission can be well described with the Compton hump, part of the reflection model, with the high-energy cut-off fixed at the maximum value in the following analysis. In Section 3.2.2, we will conduct a simultaneous $0.3-30.0 \mathrm{keV}$ broad-band analysis on both EPIC and FPM spectra.

\subsubsection{XMM-Newton EPIC data}

We first fit the $1-4 \mathrm{keV}$ energy band of the EPIC spectrum with a simple absorbed powerlaw $(\Gamma=2.8)$. The ratio plot is extended to the whole band for only illustration purpose. Fig. 5 shows the EPIC pn data/model ratio plot, indicating a strong soft excess below $1.5 \mathrm{keV}$. Two strong broad emission lines are shown in the Fe $K$ and $L$ bands, associated with the disc reflection and consistent with Ponti et al. (2010), Fabian et al. (2013), and Chiang et al. (2015).

To fit the soft excess, we separately try a phenomenological single temperature blackbody model bbody and a cut-off power-law

$2 v$ is the number of degrees of freedom. 

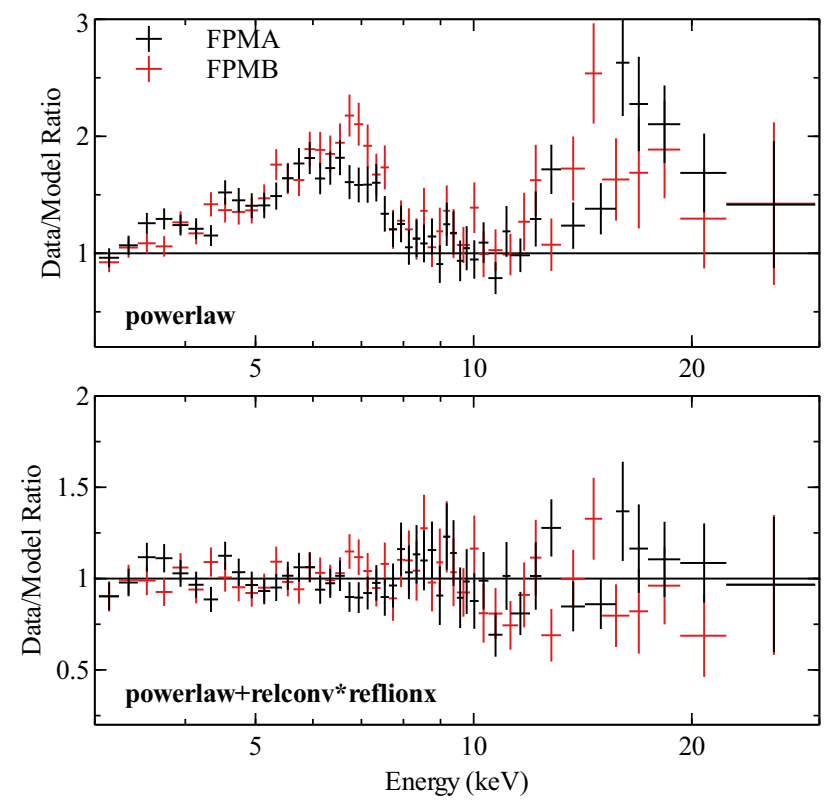

Figure 3. Top: the data/model ratio plot of two NUSTAR FPM spectra fitted with a simple Galactic absorbed power-law model. The ratio plot shows clear signatures of a disc reflection component, including a broad iron emission line and a reflection hump above $10 \mathrm{keV}$. Bottom: the ratio plot of only the FPM spectra fit with an absorbed power law plus a single relativistic disc reflection model. The total model reads constant*tbnew* (powerlaw+relconv*reflionx). More details can be found in the first column of Table 3 .

model cutoffpl. The normalization of the bbody model is defined as $L_{39} / D^{2}$, where $L_{39}$ is the source luminosity in $10^{39} \mathrm{erg} \mathrm{s}^{-1}$ and $D_{10}$ is the distance in $10 \mathrm{kpc}$. In the end of Section 3.4, we will discuss another possible interpretation of the soft excess by a new high-density disc reflection model. A distant reflection model fails to fit the broad $\mathrm{Fe} K$ emission line feature at the iron band (see the first panel of Fig. 6). So we initially use the simple and fast relativistic convolution model kdblur2, a convolution model adapted from laor (Laor 1991), which has a broken power-law emissivity profile. For further analysis, we switch to the more sophisticated relativistic kernel model relconv (Dauser et al. 2013).

We first fit the soft excess with a soft cut-off power-law model (see the second panel of Fig. 6) and a combination of a soft cut-off power-law and an additional relativistic reflection model (see the third panel of Fig. 6). In the latter case, the photon index parameters in two reflionx models are tied to that of the powerlaw model. The iron abundance parameter is tied between two reflionx models. The additional reflection model decreases the residuals of the $\mathrm{Fe} L$ emission line but the cut-off model fails to fit the spectral shape below $0.7 \mathrm{keV}$. We fit the soft excess with a blackbody model bbody instead of a soft cutoff power-law, which decreases the statistics by more than 1000 (see the fourth panel of Fig. 6). In order to fit the residuals at $<0.8 \mathrm{keV}$, we add another reflection model to fit the soft excess. It can reduce the residuals from $10 \sigma$ to $4 \sigma$ at energies below $0.6 \mathrm{keV}$ and $C$-stat by 1000 (see the fifth panel of Fig. 6). Finally, we replace kdblur2 with relconv for more accurate relativistic effects on the broad line features. A broken power-law emissivity profile is assumed. While the outer emissivity index is first fixed at 3 to meet the emissivity in flat space time, the inner emissivity index is left free to vary (see the second columns of Table 3). The inner edge of the accretion disc is assumed to be at the innermost stable circular orbit (ISCO) and the outer edge of the disc is fixed at $400 R_{\mathrm{g}}$ for simplicity. Limb-darkening effects are included in the model. The total continuum model combination now reads constant* tbnew* (relconv* (reflionx1+reflionx2) +powerlaw +bbody). This model combination provides the best fit with $C$-stat $/ v=1237.73 / 454$. The sixth panel of Fig. 6 and the lower panel of Fig. 8 show the residual and data/model ratio plots, respectively.

So far we have obtained the best-fitting continuum model, but there are still some narrow atomic features visible. For example, the absorption features in $1-2,2-4,>8 \mathrm{keV}$ band will be further discussed in Section 3.2.2 and be fitted with a photoionized absorber model xstar.

\subsection{Detailed spectral analysis}

In this section, in addition to the Fe absorption feature discussed in Parker et al. (2017b), we conduct a more detailed spectral analysis on XMM-Newton EPIC spectra first by identifying more UFO blueshifted absorption lines in the broad-band fitting and then fitting these lines with the more physical ionized absorption model xstar.

\subsubsection{Blueshifted absorption features}

As discussed in the Introduction section, a UFO from the disc of this source has been identified in this source (Parker et al. 2017a,b). Here, we present a detailed analysis and physical modelling of blueshifted Si XIV, S XVI, Nex, Mg XII absorption features in the EPIC spectra (see the sixth panel of Fig. 6 for the residual plot and Fig. 8 for the ratio plot).

We first fit the absorption feature at around $1.2 \mathrm{keV}$ in the observed frame with a Gaussian line model gauss and obtain a better fit with $C$-stat reduced by 280.79 for three-additional degrees of freedom (line energy, FWHM, and normalization). The best-fitting line energy of $\mathrm{Ne} x$ absorption line is $1.258_{-0.009}^{+0.011} \mathrm{keV}$ in the source rest frame $(1.181 \pm 0.010 \mathrm{keV}$ in the observer frame $)$ and consistent with the measurement in the RGS data $(10.0 \pm 0.5 \AA$, Parker et al. 2017b) within the measurement error. Leighly et al. (1997) also found similar absorption features at $1-1.3 \mathrm{keV}$ band in the ASCA data. Similarly to the Ne x line, we applied three more Gaussian line models for the rest of the absorption features. Parameters are given in Table 4. The blueshifted Ne X and S XVI are stronger and broader than the other two lines. In order to put limits on the strength of the ArXVII and Caxx lines found in the PCA analysis in Parker et al. (2017a), we fit the Gaussian line models to EPIC spectra with the line energy parameter fixed as 4.0 and $4.9 \mathrm{keV}$. The inclusion of the two lines does not offer significant improvement to the fit and the equivalent width is negligible (see the last two rows of Table 4).

\subsection{2 xstar Modelling}

For further study, we model the absorption features in the $1-5 \mathrm{keV}$ band (in Fig. 7) and Fe absorption feature above $8 \mathrm{keV}$ (in Fig. 5) with the physical model xstar and try to measure the overall ionization level and the averaged line-of-sight velocity of the disc outflow.

We construct custom photoionized plasma absorption models with xstar (Kallman \& Bautista 2001). The grids are calculated assuming solar abundances except for that of iron, a fixed turbulent 
Table 3. Best-fitting model parameters for NuSTAR FPM $(3.0-30.0 \mathrm{keV})$ and $X M M-N e w t o n$ EPIC $(0.3-10.0 \mathrm{keV})$ spectra. The flux is calculated by cf 1 ux in $0.3-10 \mathrm{keV}$ band and in log base 10 . The normalization of bbody model is defined as $L_{39} / D^{2}$, where $L_{39}$ is the source luminosity in $10^{39} \mathrm{erg} \mathrm{s}^{-1}$ and $D_{10}$ is the distance in $10 \mathrm{kpc}$.

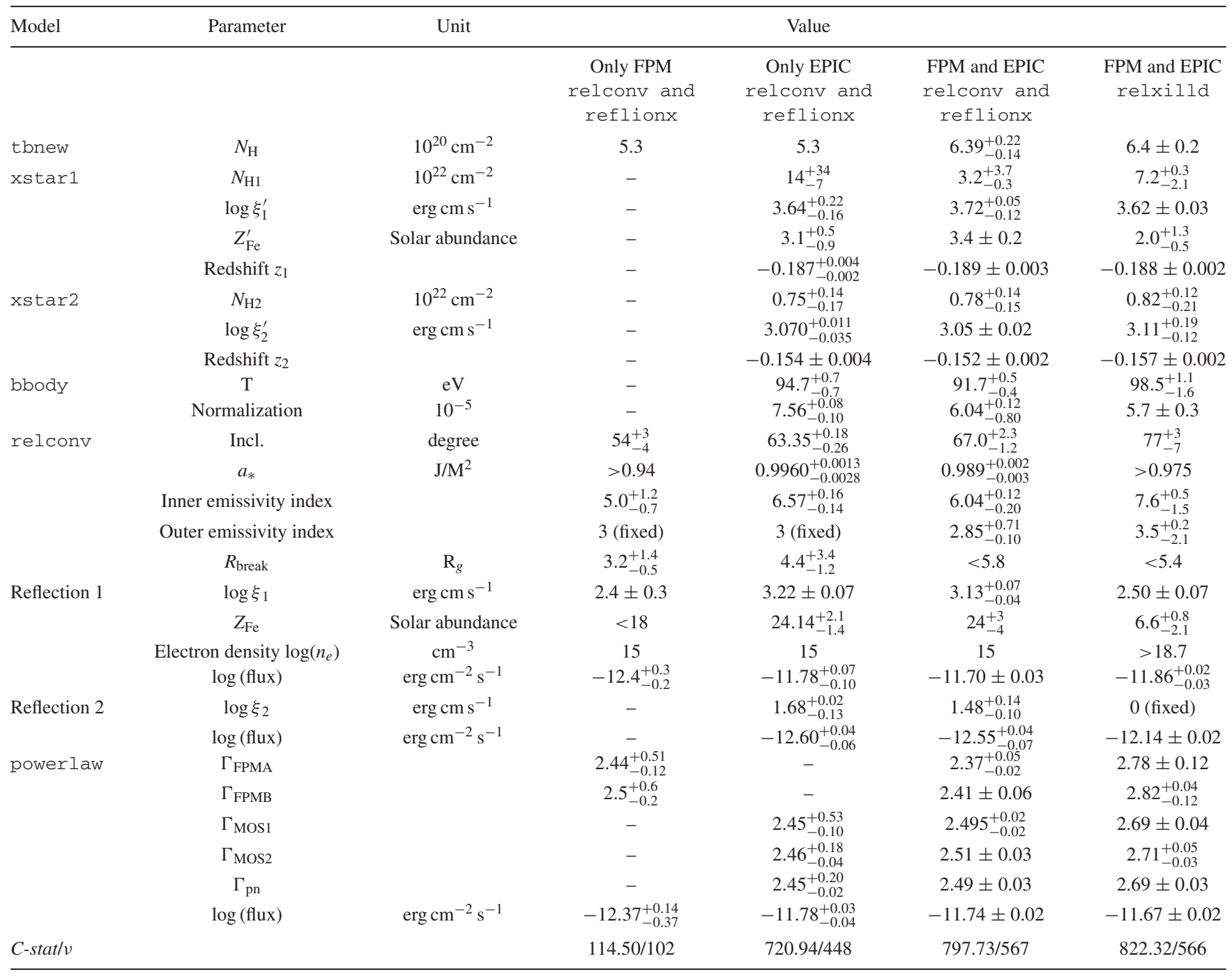

velocity of $2000 \mathrm{~km} \mathrm{~s}^{-1}$ (Parker et al. 2017b) and an ionizing luminosity of $10^{43} \mathrm{erg} \mathrm{s}^{-1}$. Free parameters are the ionization of the plasma $\left(\log \xi^{\prime}\right),{ }^{3}$ the column density $\left(N_{\mathrm{H}}\right)$, the iron abundance $\left(Z_{\mathrm{Fe}}\right)$, and the redshift $(z)$.

We first fit the EPIC spectra with only one xstar model and it reduces the $C$-stat by 99.23 . See the blue line in the top panel of Fig. 8 for the model shape. This model perfectly fits the absorption features below $5 \mathrm{keV}$, including the blueshifted $\mathrm{Ne}$ x, S XVI, Mg XII, and Si XIV absorption lines identified in Section 3.2.1. However, a single xstar model is not broad enough to fit the Fe absorption feature above $8 \mathrm{keV}$. A second xstar model is required to fit the blue wing of the $\mathrm{Fe}$ absorption line (see the red line in the top panel of Fig. 8 for the model shape). These two additional xstar models provide a significant improvement of fit by reducing the $C$-stat/v of the EPIC spectra fit by 516.79-720.94/448. The iron abundances of the two xstar models are tied together. Parameters are given in Table 3. According to the best-fitting model in the

\footnotetext{
${ }^{3}$ The prime symbol is to distinguish the ionization parameter of the outflow from that of the disc.
}

top panel of Fig. 8, the more ionized absorber $\left(\log \left(\xi_{1}^{\prime} / \mathrm{erg} \mathrm{cm} \mathrm{s}^{-1}\right)\right.$ $=3.6)$ is also more blueshifted than the less ionized absorber $\left(\log \left(\xi_{2}^{\prime} / \mathrm{erg} \mathrm{cm} \mathrm{s}^{-1}\right)=3.1\right)$. The residual plot for the fit is shown in the bottom panel of Fig. 6. Two absorbers require column density $N_{\mathrm{H} 1}=14_{-7}^{+34} \times 10^{22} \mathrm{~cm}^{-2}, N_{\mathrm{H} 2}=0.75_{-0.17}^{+0.14} \times 10^{22} \mathrm{~cm}^{-2}$, and redshift $z_{1}=-0.187_{-0.002}^{+0.004}, z_{2}=-0.154 \pm 0.004$, respectively, corresponding to line-of-sight outflowing velocity of $v=0.26,0.23 \mathrm{c}$. In the following section, we will conduct simultaneous spectral analysis on both NUSTAR and XMM-Newton data using the same model combination.

\subsection{Simultaneous spectral fitting}

Finally, we present the simultaneous fit of NuSTAR FPM and XMM-Newton EPIC spectra based on the best-fitting continuum model in Section 3.1 and the outflow model in Section 3.2.2. The total model is constant*tbnew*xstar*powerlaw+bbody+relconv* (2reflionx). The photon index parameters of different instruments are left to vary independently because this source is extremely variable and NUSTAR and XMM-Newton observations 


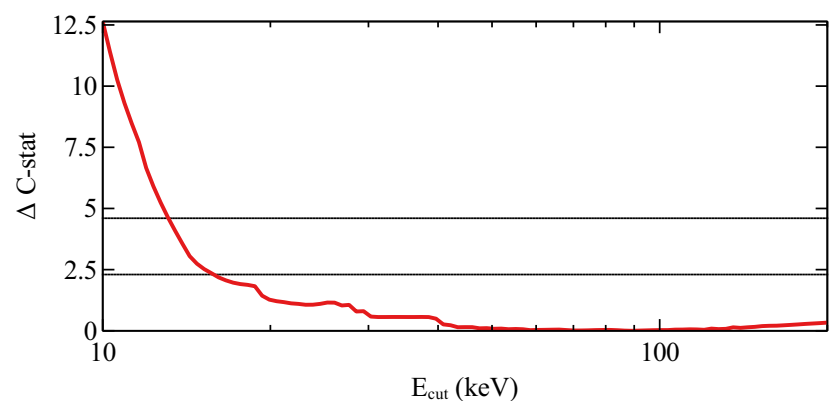

Figure 4. $\Delta C$-stat versus power-law cut-off. The red solid line is obtained by fitting the $3-30 \mathrm{keV}$ band of two NuSTAR FPM spectra. The two horizontal dotted and dashed lines are $1 \sigma$ and $2 \sigma$ measurement lines.

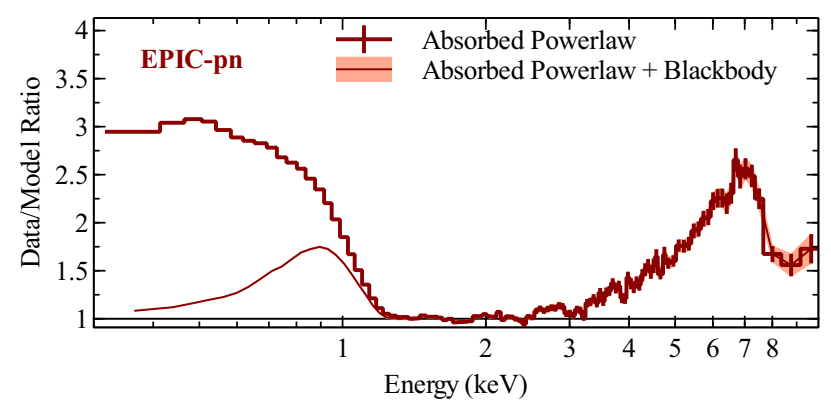

Figure 5. The data/model ratio plot for a fit of XMM-Newton EPIC-pn spectrum with a power-law model $(\Gamma=2.8)$, two simple relativistic lines and blackbody $(k T=89 \mathrm{eV})$. Shaded region: the two relativistic line models are removed to show the line shapes in the $\mathrm{Fe} K$ and $L$ bands; points with error bars: two relativistic line models and bbody are all removed to show the soft excess below $1 \mathrm{keV}$. This ratio plot shows a strong soft excess, a strong disc relativistic $\mathrm{Fe} K$ and $L$ emission lines.

are not strictly simultaneous (compare two light curves in Fig. 1). We assume a broken power-law emissivity for the disc reflection component and allow both emissivity indices to vary.

The best-fitting parameter values are listed in the third column of Table 3. The best-fitting model is shown in Fig. 9. The two xstar models have blueshift up to $z_{1}=0.189 \pm 0.003$ and $z_{2}=0.152 \pm 0.002$, corresponding to velocity of $v_{1}=0.267_{-0.003}^{+0.004} \mathrm{c}$ and $v_{2}=0.225 \pm 0.002 \mathrm{c}$, respectively, for bulk motion in the radial direction. The best-fitting column density of the outflow is $3.2_{-0.2}^{+0.7} \times 10^{22}$ and $0.78_{-0.15}^{+0.14} \times 10^{22} \mathrm{~cm}^{-2}$, which is slightly lower than the value obtained by analysing the RGS spectra $(9.5 \pm$ $0.5 \times 10^{22} \mathrm{~cm}^{-2}$ Parker et al. 2017b).

The averaged spectrum in this analysis is harder than the previous fit with the same model combination in Chiang et al. (2015) ( $\Gamma=2.71 \pm 0.02)$, which could be due to the change of the averaged temperature or optical depth of the corona through the whole observation. The best-fitting relativistic parameters indicate a fast rotating black hole $\left(0.989_{-0.003}^{+0.002}\right)$ viewed from an inclination of $67.0_{-1.2}^{+2.5} \mathrm{deg}$. The different emissivity index from the result in Chiang et al. (2015) may be due to a change of the geometry of the primary source (Wilkins et al. 2015). For example, the larger inner emissivity index means that the primary power-law photons are more concentrated on the inner disc due to the light bending effects. A very steep emissivity profile indicates a very compact primary $\mathrm{X}$-ray source.

The absorber has $Z_{\mathrm{Fe}}=3.4 \pm 0.2$ solar iron abundance while the two disc reflection components require $Z_{\mathrm{Fe}}>20$. This is not expected as the outflowing wind from the disc should share the element abundances of the disc. However, in Section 3.4 we find a
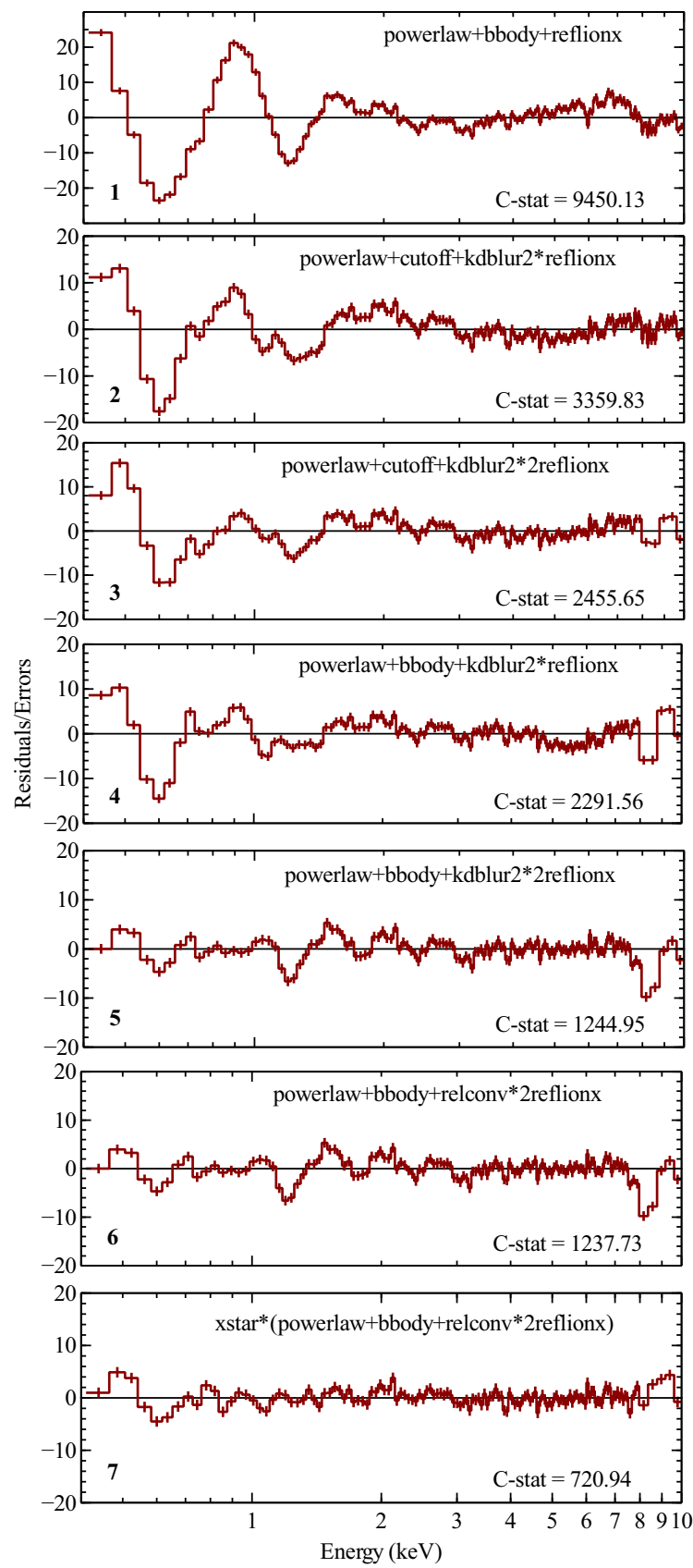

Figure 6. The residual/error plots for the different model combinations fit to the time-averaged XMM-Newton EPIC spectra (only the pn spectrum is shown here for clarity). All models are fit from 0.3 to $10.0 \mathrm{keV}$. The corresponding model and the statistics are marked in each panel. See the text for more details.

good fit with a high-density disc reflection model that only requires the disc iron abundance $Z_{\mathrm{Fe}}=6.6_{-2.1}^{+0.8}$, so can potentially solve this problem.

The best-fitting Galactic column density obtained by analysing our X-ray spectra is $6.39_{-0.14}^{+0.22} \times 10^{20} \mathrm{~cm}^{-2}$, which is slightly higher than $5.3 \times 10^{20} \mathrm{~cm}^{-2}$ predicted by Kalberla et al. (2005) but consistent with the value obtained in Willingale et al. (2013) $\left(N_{\mathrm{H}}=6.78 \times 10^{20} \mathrm{~cm}^{-2}\right)$. We will use our best-fitting Galactic column density value in the following analysis. 
Table 4. The best-fitting absorption line parameters, including line energy in observer frame, FWHM, equivalent width and the $\Delta C$-stat value. $C$-stat $/ v$ obtained by the continuum model in Section 3.1 is 1237.73/455.

\begin{tabular}{lcccc}
\hline Line & $E(\mathrm{keV})$ & FWHM $(\mathrm{keV})$ & $\mathrm{EW}(\mathrm{eV})$ & $\Delta$ C-stat \\
\hline Nex & $1.181 \pm 0.010$ & $0.24_{-0.05}^{+0.07}$ & $37.5_{-0.3}^{+0.2}$ & 280.79 \\
S XVI & $3.16 \pm 0.03$ & $0.42_{-0.05}^{+0.07}$ & $50.90_{-1.5}^{+11.0}$ & 81.75 \\
Mg XII & $1.769_{-0.03}^{+0.02}$ & $0.12_{-0.05}^{+0.07}$ & $10.0_{-0.4}^{+0.2}$ & 54.6 \\
Si XIV & $2.42 \pm 0.03$ & $0.09 \pm 0.07$ & $10.1_{-1.2}^{+4.0}$ & 28.69 \\
Ar XVII & 4.0 (fixed) & 0.001 fixed & $<2.7$ & - \\
Caxx & 4.9 (fixed) & 0.001 fixed & $<6.9$ & - \\
\hline
\end{tabular}

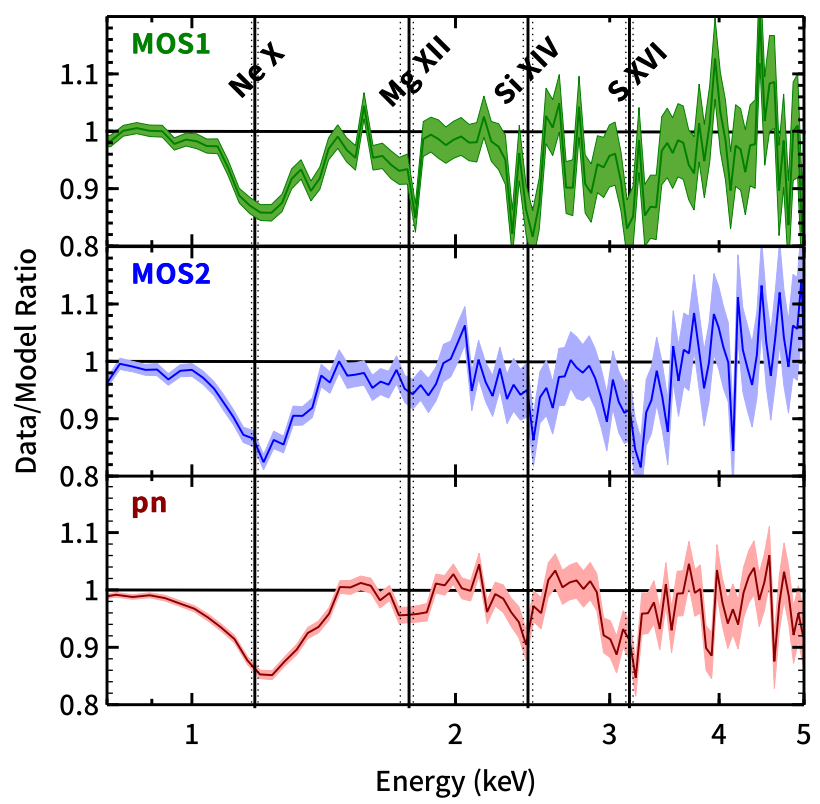

Figure 7. The data/model ratio plots for a time-averaged XMM-Newton EPIC spectral fit with the continuum model plus four additional Gaussian absorption lines. The Gaussian absorption line models in this plot are removed to show the line profiles. Blueshifted $\mathrm{Ne}$ X, Mg XII, Si XIV and S XVI absorption features are visible and labelled in $1-4 \mathrm{keV}$ band. The best-fitting line parameter values can be found in Table 4 . The vertical solid lines are the best-fitting line energy and the dotted vertical lines are the measurement errors of the corresponding line energy values.

\subsection{High electron density reflection model}

A very high iron abundance $\left(Z_{\mathrm{Fe}}=24\right)$ is required by fitting the soft excess with relativistic reflection model combination relconv*reflionx that assumes a constant electron density $n_{e}=10^{15} \mathrm{~cm}^{-3}$. In order to obtain a more reliable measurement on the disc iron abundance, we also try to fit the XMM-Newton and NuSTAR spectra with the recently developed model relxillD (García et al. 2016) that allows the electron density parameter to vary between $n_{e}=10^{15}$ and $10^{19} \mathrm{~cm}^{-3}$, while reflionx assumes $n_{e}=10^{15} \mathrm{~cm}^{-3}$. At higher $n_{e}$, the spectrum shows a higher temperature bbody shaped soft excess due to the increased influence of the free-free process on the spectrum at higher densities. Free-free absorption increasingly constrains low-energy photons, increasing the temperature of the top layer of the disc and turning the reflected emissions at energies below $1 \mathrm{keV}$ into a quasi-blackbody spectrum. García et al. (2016) show that a high electron density disc in $1 \mathrm{H}$ 0707-495 with $n_{\mathrm{e}} \approx 10^{19} \mathrm{~cm}^{-3}$ reduces the amplitude of the soft excess by 30 per cent. Tomsick et al. (2018) obtain a better fit of the

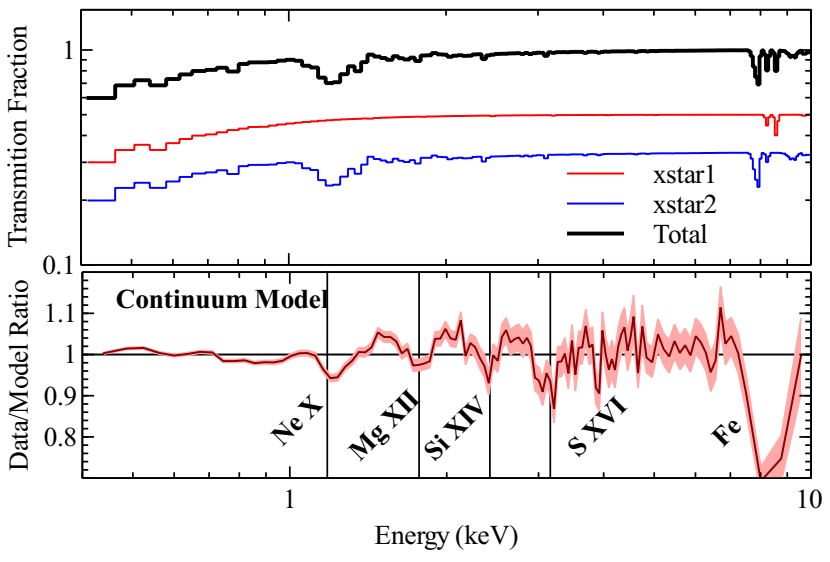

Figure 8. Top panel: Best-fitting xstar transmission model convolved with the instrumental spectral resolution after being grouped (in black) and two different ionized components (the more ionized absorber xstar1 in red and the less ionized absorber xstar2 in blue). Bottom panels: The ratio plots for time-averaged XMM-Newton EPIC pn spectra fit without xstar (only pn is shown here for clarity). All the relevant absorption lines are labelled with dashed lines.

intermediate state spectra of Cyg X-1 with the electron density as a free parameter $\left(n_{e}=\left(3.98_{-0.25}^{+0.12} \times 10^{20} \mathrm{~cm}^{-3}\right)\right.$ and only solar iron abundances.

Here, we fit the XMM-Newton EPIC and NuSTAR spectra in the $0.3-30 \mathrm{keV}$ energy band with a powerlaw, a bbody and two relativistic reflection models relxillD. The reflection fraction parameters of the two relxillD are fixed as -1 to return only the reflection components. The cut-off energy parameter $E_{\text {cut }}$ is fixed as $300 \mathrm{keV}$. The best-fitting model is shown in the top panel of Fig. 10 and it can offer a good fit with $C$-stat $/ v=$ $822.32 / 566$. One low-ionization $\left(\log \left(\xi_{1} / \mathrm{erg} \mathrm{cm} \mathrm{s}^{-1}\right)=0\right)$ and one ionized $\left(\log \left(\xi_{2} / \mathrm{erg} \mathrm{cm} \mathrm{s}^{-1}\right)=2.50 \pm 0.07\right)$ reflection component are required. The best-fitting iron abundance $Z_{\mathrm{Fe}}=6.6_{-2.1}^{+0.8}$, which is much lower than the iron abundance obtained in Section 3. The two rexillD models require disc density $n_{\mathrm{e}}>10^{18.7} \mathrm{~cm}^{-3}$ (see Fig. 11 for reference). The fit almost reaches the upper limit of the $n_{e}$ parameter in the current model. The best-fitting high-density disc reflection model is shown in Fig. 10. With such a high density, the soft band of the two reflection models has more emission than the best-fitting reflionx models shown in Fig. 9. This is still work in progress and further fits will be presented in a future paper (Jiang et al., in preparation).

\section{FLUX-RESOLVED SPECTRAL ANALYSIS}

Parker et al. (2017b) found that the $\mathrm{Fe}, \mathrm{O}$, and $\mathrm{Ne}$ absorption features are strongly flux dependent, suggesting that the UFO responds to the AGN continuum. In order to confirm whether the other absorption lines identified in Section 3.2.2 follow the same rule and probe more information on the overall spectral characteristics at different flux levels we conduct a broad-band spectral analysis on three flux resolved spectra.

We divide the EPIC pn data set into three flux levels, HF (high flux), MF (middle flux), and LF (low flux). The flux levels are chosen to have a similar number of total counts, as in Parker et al. (2017b).

We first fit all three flux-resolved spectra with the same model template discussed above, tbnew*xstar* (powerlaw + relconv* 2reflionx + bbody). As the spin measured 

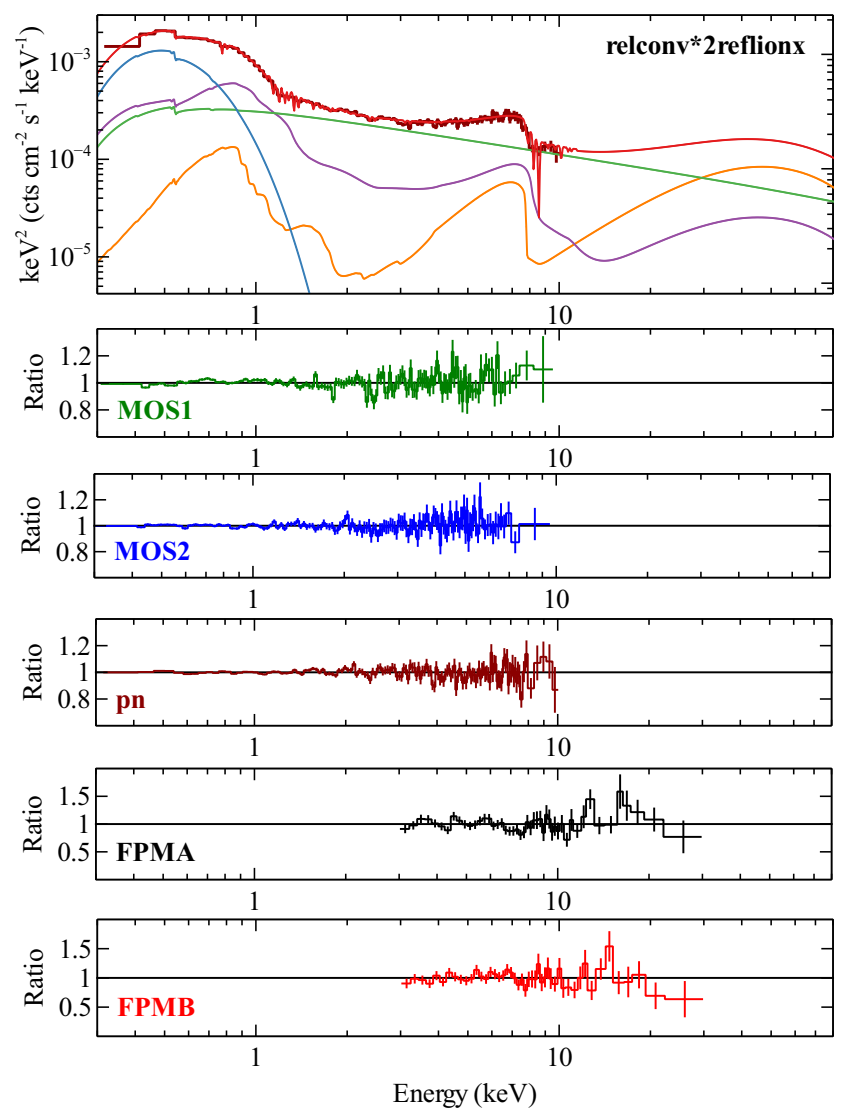

Figure 9. The best-fitting EPIC pn model for the simultaneous fit of the quasi-simultaneous XMM-Newton and NUSTAR data. The rest-frame disc reflection model used here is reflionx. Green: power-law continuum; blue: blackbody model; purple: the disc reflection component with higher ionization; yellow: the disc reflection component with lower ionization; red: the total model. The dark red with errorbars are the unfolded spectra of EPIC pn. The bottom five panels show the ratio/model plots of XMM-Newton EPIC spectra and NUSTAR FPM fitted with the best-fitting model.

in the time-averaged analysis approaches to the spin limit in General Relativity, and is consistent with previous results (Ponti et al. 2010; Fabian et al. 2013; Chiang et al. 2015; Parker et al. 2017b), we fix it at the best-fitting value obtained in the time-averaged analysis. The data/model ratio plots after removing the xstar absorption components to display the line profiles are shown in Fig. 12.

The powerlaw continuum and the corresponding disc reflection components show large different among three flux states. The bbody shows the lowest flux in the LF state, indicating a weakest soft excess. The $0.3-10 \mathrm{keV}$ band flux values of all the components increase with increasing flux levels. The coronal emission shows a softer continuum $(\Gamma=2.66$ for HF state) compared with two lower flux states (e.g. $\Gamma=2.03$ for LF state). The reflection components and the primary powerlaw continuum show higher flux at higher flux state. The primary powerlaw emission shows more than 10 times flux difference between the LF and HF states, while the flux of the reflection components only show four times difference. This can be explained by the light-bending model: the reflection component is less affected by the light bending effects as more lights are focused on to the inner area of the disc, resulting in increasing reflection fraction.
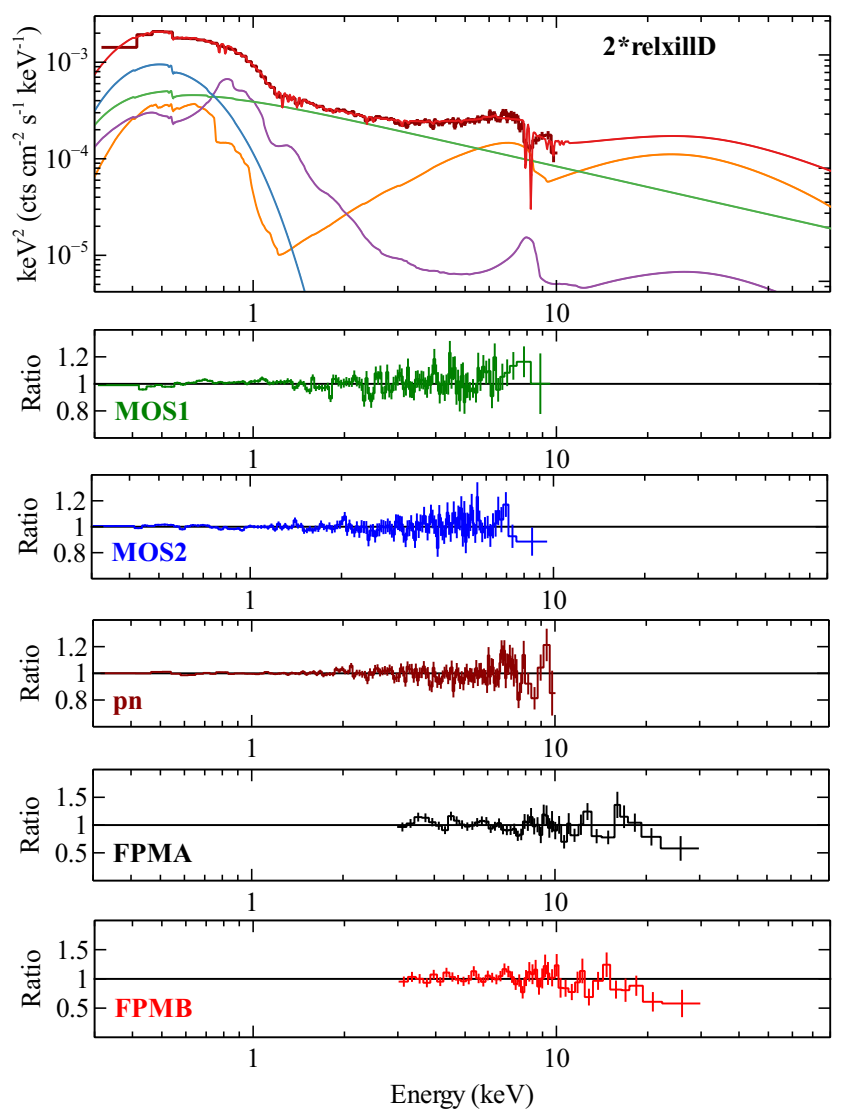

Figure 10. Same as Fig. 9. The relativistic disc reflection model used here is relxilld.

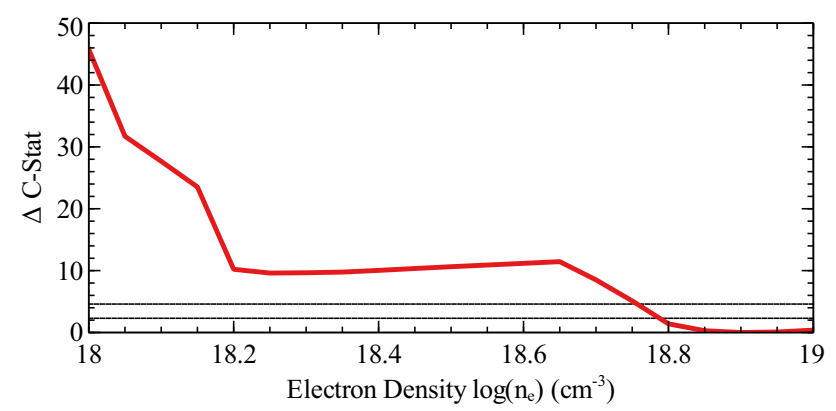

Figure 11. $\Delta C$-stat versus electron density value in logarithmic units. The two horizontal dotted and dashed lines are $1 \sigma$ and $2 \sigma$ measurement lines.

The best-fitting parameter values obtained by including the $\mathrm{xS}-$ tar models can be found in Table 5. Moreover, the blueshifted $\mathrm{Mg}$ XII, Si XIV, and S XVI absorption signatures are only visible in the lower flux states, MF and LF. To fit these absorption features, the same xstar model generated in Section 3 is applied to LF and MF spectra. xstar models significantly reduce the residuals from the continuum fit. In order to obtain the upper limit of the absorption features in HF state, we fit the HF spectrum with one xstar model $\left(\log \left(\xi^{\prime} / \mathrm{erg} \mathrm{cm} \mathrm{s}^{-1}\right) \equiv 3.0\right.$ and $\left.z \equiv-0.13\right)$. The column density we obtain is $N_{\mathrm{H}}<2 \times 10^{20} \mathrm{~cm}^{-2}$. The best-fitting outflow column density for the LF state is higher than the best-fitting value for the MF state, showing a flux-dependent outflow. 


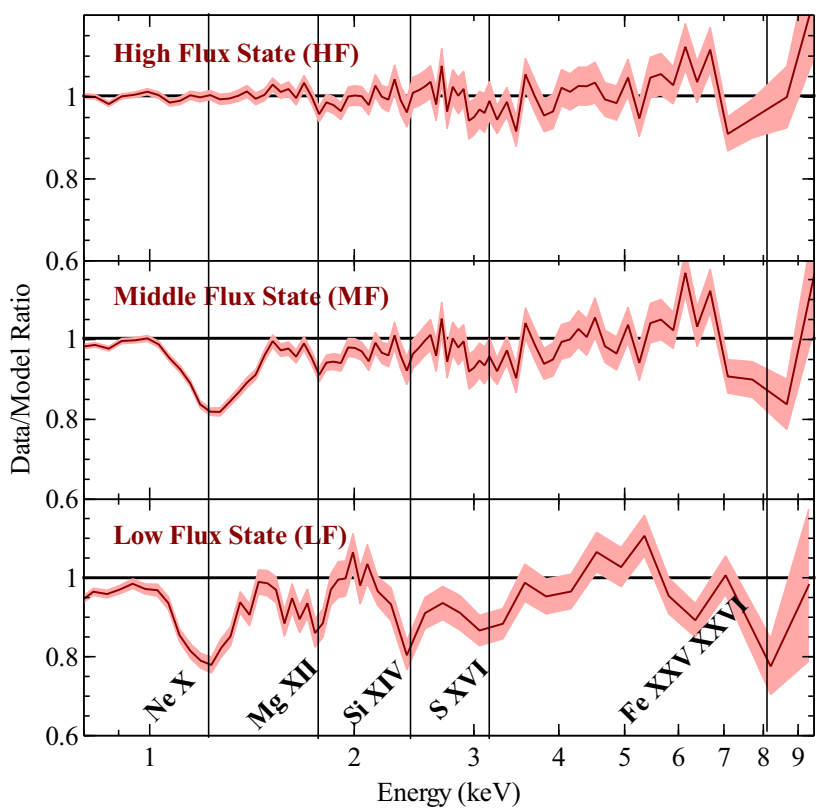

Figure 12. The ratio plot of the fits for three flux-resolved spectra, high flux (HF) state, middle flux (MF) state and low flux (LF) state to the bestfitting UFO absorbed continuum model. The xstar models are removed in this plot to show the line shapes more clearly. All the relevant blueshifted absorption features are marked with solid black lines.

\section{TIME-RESOLVED SPECTRAL ANALYSIS}

Fig. 1 shows the extreme variability of IRAS 13224-3809 in the EPIC $0.3-10 \mathrm{keV}$ band on time-scales of ks or less. In order to further study the spectral variability with respect to time and the averaged flux, we conduct spectral analysis on each of the twelve observations. A simultaneous $0.3-30 \mathrm{keV}$ broad-band spectral analysis is conducted if simultaneous NUSTAR data are available during that XMM-Newton observation. The simultaneous NUSTAR spectra are extracted according to the time coverage of the XMM-Newton observations (compare the grey-shaded region and NuSTAR combined FPM light curve Fig. 1).

All the time-resolved spectra are fitted with the same model combination as the one obtained above, with relativistic parameters and the column density fixed at the best-fitting value obtained in the time-averaged analysis. RDC and PLC are, respectively, the reflection and powerlaw continuum flux between 0.3 and $10 \mathrm{keV}$ in $\log$ scale in the unit of $\mathrm{erg} \mathrm{cm}^{-2} \mathrm{~s}^{-1}$ calculated by $\mathrm{cflux}$ model in XSPEC. The reflection fraction is simply defined as the ratio of the reflection flux over the powerlaw continuum flux. The bbody normalization is defined as $L_{39} / D_{10}^{2}$, where $L_{39}$ is the source luminosity in units of $10^{39} \mathrm{erg} \mathrm{s}^{-1}$ and $D_{10}$ is in unit of $10 \mathrm{kpc}$. All the best-fitting parameters are plotted in Fig. 13.

The following conclusions can be drawn from Fig. 13:

(i) When the blackbody flux is higher, the temperature of the blackbody tends to be higher, indicating a steeper soft excess. The temperature and luminosity of the bbody component follows the Stefan-Boltzmann $F \propto T^{4}$ relation, as found in the previous study of Chiang et al. (2015), indicating a constant emission area of the soft excess. The flux of the blackbody follows the same trend as that of the powerlaw component (see the first panel of Fig. 13).

(ii) The reflection component is highly positively correlated with the powerlaw continuum. However, as the reflection component is less variable than the powerlaw component, the reflection fraction is negatively correlated with the powerlaw flux (refer to the second and the third panels of Fig. 13). They show that the flux of the reflection components in the $0.3-10.0 \mathrm{keV}$ change by a factor of 3-4 and the flux of the powerlaw component changes by a factor of 30 . This can potentially be explained by the light-bending model: when the corona is closer to the black hole, the powerlaw flux decreases drastically due to more photons lost to the event horizon. The reflection component is less affected by the light bending affects as more light is focused on to the inner disc, resulting in an increasing reflection fraction.

(iii) From the Spearman's rank correlation $(\mathrm{SRCC}=-0.634$, $P$-value $=0.27$ using $t$-distribution), we find that the inner emis-

Table 5. Best-fitting parameter values for three flux-resolved spectra. The units of the parameters are defined the same as in Table 3 .

\begin{tabular}{|c|c|c|c|c|}
\hline Model & Parameter & High flux (HF) & Middle flux (MF) & Low flux (LF) \\
\hline \multirow[t]{3}{*}{ xstar1 } & $N_{\mathrm{H} 1}\left(10^{22} \mathrm{~cm}^{-2}\right)$ & - & $<1.0$ & $4.2_{-2.1}^{+1.3}$ \\
\hline & $\log \xi_{1}^{\prime}\left(\log \left(\mathrm{erg} \mathrm{cm} \mathrm{s}^{-1}\right)\right)$ & - & $4.3_{-0.7}^{+0.8}$ & $3.7_{-0.5}^{+0.2}$ \\
\hline & redshift $z_{1}$ & - & $-0.170_{-0.005}^{+0.002}$ & $-0.175 \pm 0.006$ \\
\hline \multirow[t]{3}{*}{ xstar2 } & $N_{\mathrm{H} 2}\left(10^{22} \mathrm{~cm}^{-2}\right)$ & - & $0.6 \pm 0.4$ & $13.7 \pm 1.4$ \\
\hline & $\log \xi_{2}^{\prime}\left(\log \left(\mathrm{erg} \mathrm{cm} \mathrm{s}^{-1}\right)\right)$ & - & $3.07 \pm 0.07$ & $3.0_{-0.3}^{+0.4}$ \\
\hline & redshift $z_{2}$ & - & $-0.143_{-0.02}^{+0.05}$ & $-0.132 \pm 0.007$ \\
\hline \multirow[t]{2}{*}{ bbody } & $k T(\mathrm{eV})$ & $93.7_{-1.2}^{+0.2}$ & $91.3_{-1.1}^{+0.2}$ & $92.3_{-0.5}^{+0.3}$ \\
\hline & $\operatorname{norm}\left(10^{-5}\right)$ & $9.7 \pm 0.3$ & $8.15_{-0.20}^{+0.12}$ & $3.63_{-0.11}^{+0.07}$ \\
\hline \multirow[t]{2}{*}{ reclonv } & Inner emissivity index & $5.2_{-0.3}^{+0.4}$ & $6.3_{-2.0}^{+1.2}$ & $6.0_{-3.5}^{+1.0}$ \\
\hline & $R\left(R_{g}\right)$ & $<9$ & $<8$ & $69_{-56}^{+11}$ \\
\hline \multirow[t]{2}{*}{ reflionx1 } & $\log \xi_{1}\left(\mathrm{erg} \mathrm{cm} \mathrm{s}^{-1}\right)$ & $3.36_{-0.04}^{+0.02}$ & $3.24_{-0.06}^{+0.19}$ & $2.70_{-0.12}^{+0.17}$ \\
\hline & $\log$ (flux) $\left(\mathrm{erg} \mathrm{cm}^{-1} \mathrm{~s}^{-1}\right)$ & $-11.41 \pm 0.03$ & $-11.84_{-0.09}^{+0.06}$ & $-12.58_{-0.04}^{+0.08}$ \\
\hline \multirow[t]{2}{*}{ reflionx2 } & $\log \xi_{2}\left(\mathrm{erg} \mathrm{cm} \mathrm{s}^{-1}\right)$ & $1.53_{-17}^{+0.13}$ & $2.12_{-0.08}^{+0.04}$ & $1.80_{-0.21}^{+0.14}$ \\
\hline & $\log$ (flux) $\left(\mathrm{erg} \mathrm{cm}^{-1} \mathrm{~s}^{-1}\right)$ & $-12.35_{-0.15}^{+0.12}$ & $-12.45_{-0.12}^{+0.20}$ & $-12.77 \pm 0.08$ \\
\hline \multirow[t]{3}{*}{ powerlaw } & $\Gamma_{\mathrm{pn}}$ & $2.66 \pm 0.04$ & $2.31_{-0.02}^{+0.04}$ & $2.03 \pm 0.04$ \\
\hline & $\log ($ flux $)\left(\operatorname{erg~cm}{ }^{-1} \mathrm{~s}^{-1}\right)$ & $-11.33_{-0.04}^{+0.03}$ & $-11.90_{-0.04}^{+0.07}$ & $-12.28_{-0.03}^{+0.04}$ \\
\hline & $C$-stat $/ v$ & $166.79 / 135$ & $157.46 / 120$ & $141.58 / 119$ \\
\hline
\end{tabular}



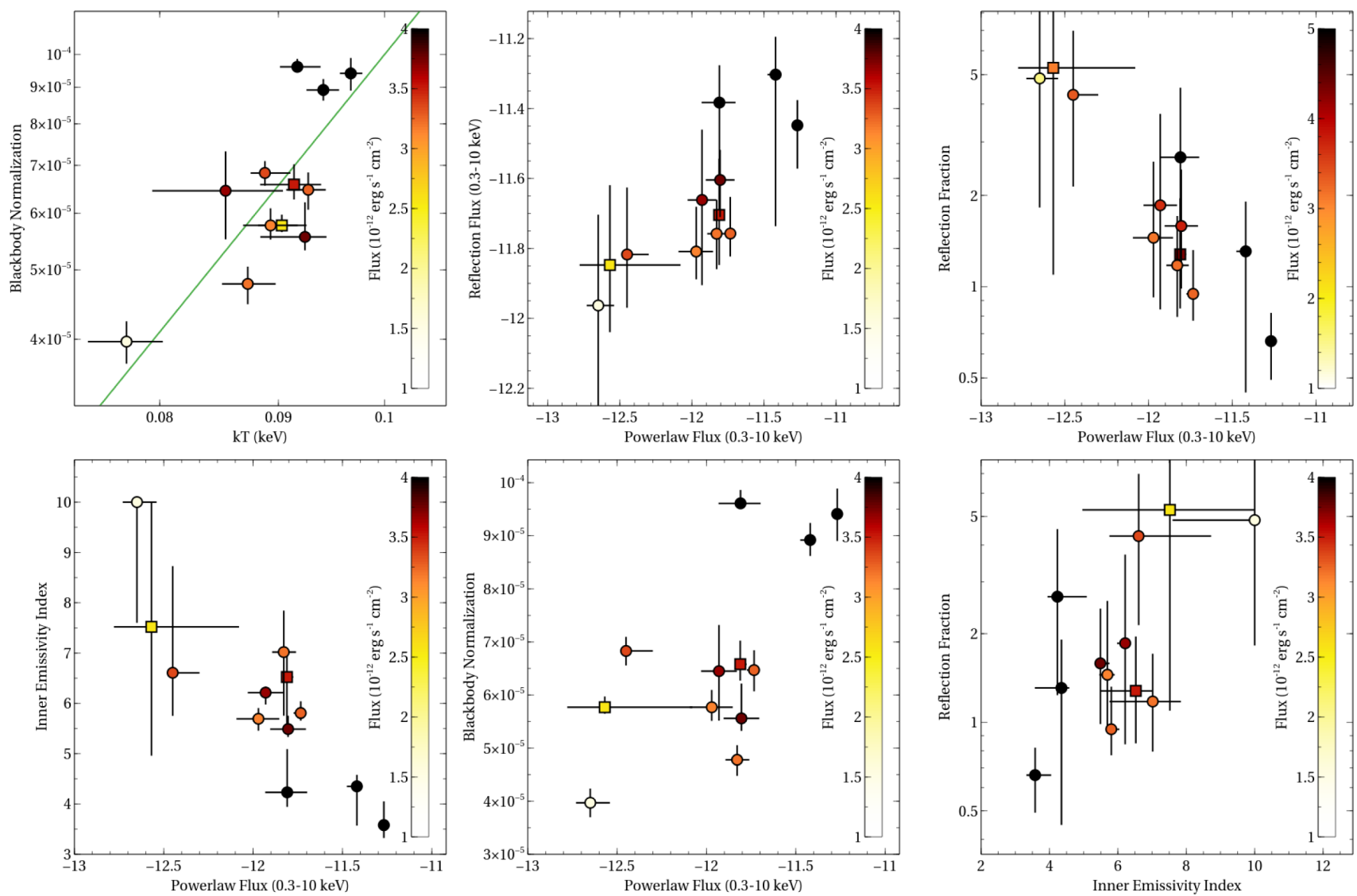

Figure 13. Time-resolved spectral analysis results. PLC and RDC are, respectively, the powerlaw continuum and the reflection flux in log scale calculated by cflux between 0.3 and $10 \mathrm{keV}$. The reflection fraction is defined as the ratio of the reflection flux to the powerlaw continuum flux in that band. The Stefan-Boltzmann relation $F \propto T^{4}$ is plotted with a green line in the first panel for reference. The emissivity index here is the inner emissivity index, while the outer emissivity index is fixed at 3 . The colour bar of the plot symbol indicates the average flux in the corresponding time slice. The best-fitting values obtained by analysing two XMM-Newton observations without simultaneous NUSTAR data are marked in squares.

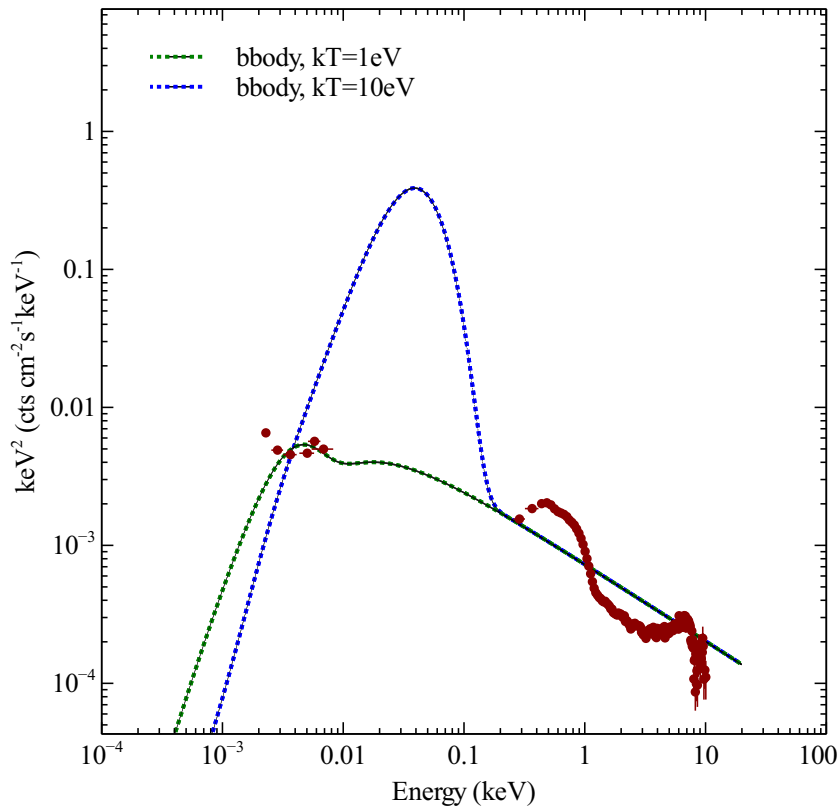

Figure 14. Continuum fitting with bbody+powerlaw to IRAS 13224-3809 XMM-Newton and Swift (uvot) data. The blue and green dotted lines are the best-fitting phenomenological continuum models with $k T$ $=1,10 \mathrm{eV}$. See the text for more details. sivity index and the powerlaw flux have a weak negative correlation. This can also be explained by a change of primary source height in the lamp-post geometry as in the point above. The inner emissivity tends to be higher when the source is close to the black hole and the primary photons are more concentrated to the inner area of the disc due to the light-bending effects. The primary powerlaw component is weaker, caused by the loss of the primary photons to the central black hole (refer to the fourth panel of Fig. 13).

(iv) In Fig. 1, we show the best-fitting powerlaw photon index to compare with the light curve. There is a positive correlation between the source brightness in 0.3 and $10.0 \mathrm{keV}$ band and the powerlaw photon index. The brighter the source is, the softer the power law is.

\section{DISCUSSION}

\subsection{Overall bolometric luminosity estimation}

In this section, we calculate the bolometric luminosity in three ways. We first apply a simple bolometric correction to the observed flux in the $2-10 \mathrm{keV}$ band. Secondly, we apply the linear relation between the spectral index and the Eddington ratio found in Brightman et al. (2013). Thirdly, a continuum fitting method is applied to the UV/optical-X-ray band first with a phenomenological model (bbody+powerlaw). As the mass is uncertain, we leave a factor of $10^{7} \mathrm{M}_{\odot} / M$ in our estimates of Eddington fraction. 
First, the best-fitting model for time-averaged spectra in Table 3 shows the source has flux of $6.87 \times 10^{-13} \mathrm{erg} \mathrm{cm}^{-2} \mathrm{~s}^{-1}$ in the 2$10 \mathrm{keV}$ band. Given the measurement that the luminosity distance of IRAS $13224-3809$ is $288 \mathrm{Mpc}, L_{2-10 \mathrm{keV}}=6.82 \times 10^{42} \mathrm{erg} \mathrm{s}^{-1}$. $L_{\text {Edd }}$ for central object with mass $10^{7} \mathrm{M}_{\odot}$ is $12.6 \times 10^{44} \mathrm{erg} \mathrm{s}^{-1}$. According to the estimation of the bolometric correction given for the $2-10 \mathrm{keV}$ flux for NLS1 (see the top panel of Fig. 12 in Vasudevan \& Fabian 2007), the overall bolometric luminosity $L_{\text {bol }}^{\text {ave }}$ $=\kappa_{2-10 \mathrm{keV}} \times L_{2-10 \mathrm{keV}} \approx 50 \times 6.82 \times 10^{42} \mathrm{erg} \mathrm{s}^{-1}=3.4 \times 10^{44} \mathrm{erg}$ $\mathrm{s}^{-1}$. The best-fitting model flux for the flux peak spectrum is around $9.28 \times 10^{-13} \mathrm{erg} \mathrm{cm}^{-2} \mathrm{~s}^{-1}$ between 2 and $10 \mathrm{keV}$, corresponding to bolometric luminosity $L_{\text {bol }}^{\text {peak }}=4.65 \times 10^{44} \mathrm{erg} \mathrm{s}^{-1}$. This means that the source remains sub-Eddington both on average $\left(0.27 \frac{10^{7} \mathrm{M}_{\odot}}{M} L_{\text {Edd }}\right)$ and at peaks $\left(0.37 \frac{10^{7} \mathrm{M} \odot}{M} L_{\text {Edd }}\right)$ according to the calculation of only the X-ray band. This result is roughly consistent with Sani et al. (2010).

Secondly, according to the estimation of the bolometric ratio given by the powerlaw photon index, $\lambda_{\text {Edd }}=69.78$ for the peak spectra $(\Gamma=2.86)$ and $\lambda_{\text {Edd }}=4.22$ on average $(\Gamma=2.47)$ by using the correlation $\Gamma=(0.32 \pm 0.05) \log \lambda_{\text {Edd }}+(2.27 \pm 0.06)$ (Brightman et al. 2013). The extremely high Eddington ratio obtained for the flux peak, however, is very uncertain, because the samples in Brightman et al. (2013) do not have sources with the primary continuum softer than $\Gamma=2.2$.

Thirdly, we try to estimate the accretion rate by fitting the SED with phenomenological model. The optical monitor (OM) on XMMNewton was operated with only one filter during the observing campaign. So we extract the photometry data from simultaneous Swift (uvot) observations (Buisson et al. 2018). We used a circular source region of 5 arcsec radius and circular background region of 15 arcsec radius from a nearby source free area. UV fluxes have been corrected for Galactic reddening $E(B-V)=0.06$. We first try to fit with the phenomenological continuum model bbody+power law. The bbody model is for the disc thermal component and the powerlaw is for the primary continuum in the harder band. We fix the photon index of the power law as 2.5 , the same as we obtained in the time-averaged spectral analysis. The temperature of the thermal component is fixed at 1 and $10 \mathrm{eV}$ to obtain upper limit and lower limits on the broad-band luminosity. The best-fit models are shown in Fig. 14. The fit with $k T=1 \mathrm{eV}$ gives a total model luminosity between 1 and $10^{4} \mathrm{eV}$ around $4 \times 10^{-11} \mathrm{erg} \mathrm{cm}^{-2} \mathrm{~s}^{-1}$, corresponding to $0.3 \frac{10^{7} \mathrm{M}_{\odot}}{M} L_{\mathrm{Edd}}$, and the fit with $k T=10 \mathrm{eV}$ gives $4 \times 10^{-10} \mathrm{erg}$ $\mathrm{cm}^{-2} \mathrm{~s}^{-1}$, corresponding to $3.0 \frac{10^{7} \mathrm{M}_{\odot}}{M} L_{\text {Edd }}$. Therefore, the estimation of the bolometric luminosity by the continuum fitting with a phenomenological continuum model is $0.3-3 \frac{10^{7} \mathrm{M} \odot}{M} L_{\mathrm{Edd}}$. This measurement is consistent with the results obtained by Ponti et al. (2010), where they fit the UV and $2-10 \mathrm{keV} X M M-N e w t o n$ data with diskpn and estimated $\lambda_{\text {Edd }} \approx 1$ for a $10^{7} \mathrm{M}_{\odot}$.

The estimation of the bolometric luminosity suggests that the source is close to the Eddington limit and may suffer accretion instability causing extreme X-ray variability. This may also be relevant to the launching of the UFO and the rapid X-ray variability (e.g. Done \& Jin 2016). It is generally thought that the most powerful outflows are launched near to the Eddington limit, and powerful outflows are observed in the ultraluminous X-ray sources (Pinto, Middleton \& Fabian 2016; Walton et al. 2016; Pinto et al. 2017; Kosec et al. 2018), at least some of which are superEddington neutron stars (Bachetti et al. 2014; Fürst et al. 2016; Israel et al. 2017a,b). Similarly, Leighly (2001) also found there are more high ionization emission lines observed in the UV band that are dominated by wind emission in the objects close the Eddington limit.

\subsection{Soft excess}

In our analysis, the soft excess can be fit very well with a simple bbody component and an additional relativistic reflection component. The bbody temperature and flux follow $F \propto T^{4}$ relation, which indicate a constant emission area for the soft excess. Chiang et al. (2015) discussed possible explanations of the soft excess as reprocessing of both the coronal emission and the reflection. Strong gravitational effects can cause the thermal and reflected disc photons to return to the disc surface (Cunningham 1976). The innermost area of the disc is thus heated sufficiently to emit blackbody radiation in the soft band.

In Section 3.4, we explore the possibility of the soft excess as part of the disc reflection. relxilld is an extended version of relxill and models a relativistic reflection spectrum from an accretion disc with the electron density on the surface of the disc allowed to vary freely (reflionx assumes $n_{\mathrm{e}} \equiv 10^{15} \mathrm{~cm}^{-3}$ ). We successfully fit the EPIC spectra in the $0.3-10 \mathrm{keV}$ energy band by replacing reflionx with relxillD models. The fit favours reflionx over relxillD with smaller value of $C$-stat. But the best-fitting iron abundance $Z_{\mathrm{Fe}}=6$, which is much lower than the iron abundance obtained in Section 3. We have obtained a strong constrain on the lower limit of the electron density $n_{\mathrm{e}}>10^{18.7} \mathrm{~cm}^{-3}$ of the top layer of the disc by fitting the soft excess with a combination of high-density reflection models and a phenomenological blackbody-shaped model. The two xstar models are consistent with the ones obtained in Section 3.2.2.

The high-density disc reflection model proposed in García et al. (2016) is based on an extended model of the standard accretion disc. The electron density at high accretion rates is $n_{e} \dot{m}^{2} \propto(1-f)^{-3}$, where $f$ is the fraction of power released by the disc on to the corona and $\dot{m}$ is the accretion rate in Eddington unit (Svensson \& Zdziarski 1994). If 90 per cent of the disc power is taken by the corona $(f=0.9), n_{e} \dot{m}^{2} \approx 10^{19}-10^{18} \mathrm{~cm}^{-3}$ for black hole masses of $10^{6}-10^{7} \mathrm{M}_{\odot}$ (see fig. 1 of García et al. 2016). At the electron density $n_{e}$ as high as $10^{18.7} \mathrm{~cm}^{-3}$, the reflection spectrum shows a higher temperature bbody shaped soft excess. This is due to the increased influence of the free-free process on the spectrum at higher densities. Free-free absorption increasingly constrains low-energy photons, forcing the surface temperature of the reflecting material to rise and turning the reflected emissions at energies below $1 \mathrm{keV}$ into a quasi-blackbody spectrum. The continuum shape can also depend on the density of the disc. The powerlaw continuum is usually interpreted as the thermal Comptonization of the seed photons from the disc. The spectral index of this continuum has a positive correlation with the corona temperature and the optical depth $\tau$ (Zdziarski, Johnson \& Magdziarz 1996). In our time-resolved spectral analysis result, the powerlaw continuum is softer when the bbody temperature is higher. The change of powerlaw hardness is caused by either by a change of the temperature of the primary source or a change of geometry. Since the former factor increases when $n_{e}$ increases, $\tau$, the geometry-dependent parameter, must change and the optical depth must be lower when the source is softer and brighter.

The current version of relxilld still requires improvement. For instance, more element abundances are expected to be higher than the solar level as well as iron. This is still work in progress and further fits will be presented in a future paper (Jiang et al., in preparation). 


\subsection{Disc reflection}

To better model a turbulent accretion disc, two reflection components with different ionizations are used, one with a moderate ionization of $\log \left(\xi_{1} / \mathrm{erg} \mathrm{cm} \mathrm{s}^{-1}\right)=3.13$ and one with a low ionization $\log \left(\xi_{2} / \mathrm{erg} \mathrm{cm} \mathrm{s}^{-1}\right)=1.48$. The current model requires overabundant iron, while the other elements are assumed to be solar due to the limitations of the reflionx model we use for analysis. However, as discussed above the need for a very high iron abundance and additional bbody can be potentially reduced by replacing the reflionx model with extended version of relxilld with higher disc density (discussed in Section 6.2). The disc iron abundance is $Z_{\mathrm{Fe}}=6.6_{-2.1}^{+0.8}$ with relxillD and more consistent with the iron abundance of the UFO we obtain by fitting the absorption lines with xstar. The high iron abundance, even when fitted with highdensity disc reflection model, indicates that the other elements might be more abundant than solar. Wang et al. (2012) presented a strong correlation between the outflow strength in quasars, measured by the blueshift and asymmetry index (BAI), and the metallicity, measured by Si Iv O IV/C Iv, based on the quasar samples built in the Sloan Digital Sky Survey. For example, a significantly higher metallicity $(Z>5)$ is indicated for quasars with BAI $>0.7$. The metallicity may play an important role and be connected with the quasar outflow.

Various authors (Martocchia \& Matt 1996; Martocchia, Karas \& Matt 2000; Wilkins \& Fabian 2012; Dauser et al. 2013) have shown that for simple coronal geometries the radial emissivity index decreases very sharply with source height in the most inner area of the disc (as low as $q=1$ when the source height is up to $100 R_{g}$ ). It tends to approximate as $q=3$ in the outer area of the disc where the spacetime can be approximated to be flat. This effect reduces sharply with the source height. When the source height is $3 R_{g}$, the emissivity profile is well approximated by a broken power law (outer emissivity index $q=3$ ) with a very low break radius. A high inner emissivity index and a low break radius as in IRAS 13224-3809 indicates a very small source height ( $<2 R_{g}$ for instance).

IRAS 13224-3809 was observed by XMM-Newton for $500 \mathrm{ks}$ in 2011. Chiang et al. (2015) estimated the spectral variability during the observation and obtained a steeper emissivity profile for the time-averaged spectra (inner emissivity index $q_{1}>9$, outer emissivity index $q_{2}=3.4_{-0.2}^{+0.3}$ and $R_{\text {break }}=2.1 \pm 0.1 R_{g}$ ) than we find. This could be caused by differences in the averaged source height in two observations. Moreover, the new campaign captures stronger flux peaks $\left(12 \mathrm{cts} \mathrm{s}^{-1}\right)$ than Chiang et al. (2015) $\left(8 \mathrm{cts} \mathrm{s}^{-1}\right)$, which is consistent with a higher source and therefore less extreme emissivity profile.

The second and the third panels of Fig. 13 show that the powerlaw continuum is more variable than the reflection component. The reflection fraction has an inverse relation with the powerlaw flux though the reflection component flux follows the same trend with the powerlaw flux. The light-bending solution (Miniutti et al. 2003) is potentially a good explanation for this: when the corona is closer to the central black hole the trajectories of more photons will be bent towards the black hole and more primary continuum photons will be lost. The reflection component is however less affected by the light bending effects, as more light is focused on to the inner disc, resulting in an increasing reflection fraction. Similar results have been found in other sources, such as MCG-6 - 30-15 (Miniutti et al. 2003; Vaughan \& Fabian 2004), NGC 3783 (Reis et al. 2012), and XRB XTE J1650-500 (Rossi et al. 2005; Reis et al. 2013), where the variability is dominated by the powerlaw continuum. An extreme case is the NUSTAR observation on Mrk 335 in 2013.
Parker et al. (2014) found the reflection fraction decreases sharply with the increasing flux. The low-flux spectra of Mrk 335 are well described by only disc reflection model and indicates extreme light bending effects happening within $2 R_{g}$. The anticorrelation between the inner emissivity index and the flux of the powerlaw continuum (the fourth panel of Fig. 13) in IRAS 13224-3809 also supports this interpretation. When the source is closer to the central black hole, the flux of the primary continuum decreases due to stronger light bending and the emissivity index is higher due to photons being focused on to the inner disc.

\subsection{Ultra fast outflow}

The combined spectral analysis of the stacked NuSTAR and $X M M-N e w t o n$ spectra shows two relativistic outflowing absorbers in the source. The more ionized absorber $\left(\log \left(\xi_{1}^{\prime} / \mathrm{erg} \mathrm{cm} \mathrm{s}^{-1}\right)=\right.$ $\left.3.72_{-0.12}^{+0.05}\right)$ has a higher blueshift $\left(z_{1}=0.189 \pm 0.003\right.$, corresponding to line-of-sight velocity $v_{1}=0.267_{-0.03}^{+0.04} \mathrm{c}$ ), while the less ionized absorber $\left(\log \left(\xi_{2}^{\prime} / \mathrm{erg} \mathrm{cm} \mathrm{s}^{-1}\right)=3.05 \pm 0.02\right)$ has a lower blueshift $\left(z_{2}=0.152 \pm 0.002\right.$, corresponding to line-of-sight velocity $v_{2}=0.225 \pm 0.002 \mathrm{c}$ ). We note that a similar UFO is found in 1H0707-495: Dauser et al. (2012) identified blueshifted narrow features at $2-5 \mathrm{keV}$ band from $\mathrm{H}$-like ions $(\mathrm{Si}, \mathrm{S}, \mathrm{Ca}$ ) in 1H0707-495 XMM-Newton spectra as ultra-fast wind absorption features. The ionization of the disc wind in 1H0707-495 has small fluctuations $\left(\log \left(\xi / \mathrm{erg} \mathrm{cm} \mathrm{s}^{-1}\right) \approx 3.5\right)$, but the velocity has a difference of $0.07 \mathrm{c}$ between different observations. Hagino et al. (2016) also found evidence for an Fe absorption feature at 7.1-7.5 keV, with a velocity of $0.18 \mathrm{c}$, also consistent with the velocity found by Dauser et al. (2012) ( $v=0.11-0.18 \mathrm{c})$.

As found in Parker et al. (2017b), the inclusion of the UFO absorption lines does not have any significant impact on the measured reflection parameters. Similarly, in 1H0707-495, Dauser et al. (2012) find no large difference on the relativistic parameters, such as the corona height in the lamp-post scenario, the black hole spin and the viewing angle, after including the wind component. This is contrary to the results of Hagino et al. (2016), who found that the relativistic blurring parameters for $1 \mathrm{H} \mathrm{0707-495}$ were less extreme when UFO absorption was taken into account. This difference is likely due to the much higher data quality and broader energy band used here, where we consider stacked spectra with very high signal to noise, whereas Hagino et al. (2016) examine individual observations and largely limit their analysis to the $2-10 \mathrm{keV}$ band.

The lower ionization absorber is mainly used to fit the low-energy absorption lines below $5 \mathrm{keV}$ such as $\mathrm{Ne} x$ line and the red wing of the Fe absorption line, while the more ionized absorber is to fit the blue wing of the iron absorption above $8 \mathrm{keV}$. Parker et al. (2017b) and Pinto et al. (2018) fit the UFO absorption in the RGS and high-energy EPIC-pn spectra with a single absorber, with a velocity intermediate between the two we find here. The significant improvement that we find for fitting with two zones instead of one is likely an indication that there is structure or stratification of the UFO material, which we discuss below. The increasing level of ionization increases the rest-frame energy of the atomic feature lines and removes some of them as well (compare the red and blue lines in Fig. 8 for reference). Thanks to the high signal/noise of the XMM-Newton EPIC cameras in the soft band, in addition to the iron absorption feature at $8.1 \mathrm{keV}$ found in the EPIC-pn spectrum (Parker et al. 2017b) we have identified Ne x (equivalent width $37.5 \mathrm{eV}$ ), S XVI (equivalent width $50.90 \mathrm{eV}$ ), $\mathrm{Mg}$ XII (equivalent width $10.0 \mathrm{eV}$ ), and Si XIV (equivalent width $10.1 \mathrm{eV}$ ) absorption lines (see Fig. 7 for reference). When the source is at high 
fluxes, the absorption features are weaker, consistent with the wind being more photoionized due to more photons emitted from the continuum source to the outflowing wind (see Fig. 12).

There are several different scenarios that could explain the observed outflow properties. An accelerating wind model was proposed in Murray et al. (1995) where the outflowing velocity increases along the outflow stream line. However, our data show that the faster absorber has higher photon ionization, which is contrary to the expectation in this model. Another possible interpretation is that the highly ionized layer lies inside a low ionized layer of the wind, and is more exposed to the continuum photons. This model was also used to explain the properties of the UV data of NGC 5548 in (Elvis 2000). Alternatively, Gallo \& Fabian (2011) proposed that the absorption features result from ionized materials corotating with the disc in a surface layer. A different line of sight through this layer, caused by changes in coronal geometry, can result in a different observed optical depth and therefore different absorption feature. Such a model has been successfully applied to PG 1211+143 (Gallo \& Fabian 2013). According to this model, the coexistence of a more ionized faster absorber and a less ionized slower absorber in our analysis can be interpreted as a layer in the inner disc that is more photoionized and faster than the layer further out.

These two absorbers do not show significant changes with time during the observation. It indicates a relatively constant outflow from the disc in the time-scale of kiloseconds, confirming the result of Parker et al. (2017b) and Pinto et al. (2018), where we show that the Fe Xxv/Xxvi absorption feature has been approximately constant since 2011. This is interesting, as the UFO in PDS 456, which has a much larger $M_{\mathrm{BH}} \approx 10^{9} \mathrm{M}_{\odot}$, shows significant changes in velocity during observations (e.g. Matzeu et al. 2016, 2017). Why this would not be seen in the far more rapidly variable AGN IRAS 13224-3809 is not obvious. It is possible that there are velocity changes on timescales that we cannot resolve, so we see averaged (and therefore broadened) absorption lines, or it could instead be that there is an intrinsic difference between these outflows.

\section{CONCLUSIONS}

We fit the spectra from the $1.5 \mathrm{Ms} X M M-N e w t o n$ and $500 \mathrm{ks} \mathrm{NuS-}$ TAR observing campaign on the extreme NLS1 IRAS 13224-3809 with physical broad-band models. We analyse stacked spectra, as well as flux-resolved and time-resolved spectra. Our main results are as follows:

(i) IRAS $13224-3809$ is the most extremely variable AGN. The 0.3-10.0 keV band light curve shows rapid variability on timescale down to kiloseconds, and we find a peak flux 100 times the lowest level.

(ii) Two reflection components with different ionization $\left(\log \left(\xi_{1,2} / \mathrm{erg} \mathrm{cm} \mathrm{s}^{-1}\right)=3.13,1.48\right)$ are required to fit the stacked spectra, as found by previous authors.

(iii) The emissivity profile of the reflected emission steepens as the powerlaw flux drops. This can be explained by a variable primary source height in the lamp post scenario.

(iv) The variable blackbody component, used to fit some of the soft excess, follows the $F \propto T^{4}$ relation, indicating a constant emission area in the soft band.

(v) Four blueshifted absorption lines ( $\mathrm{Nex}, \mathrm{S}$ xVI, $\mathrm{Mg}$ XII, and $\mathrm{Si}$ XIV) are detected in the stacked EPIC spectra. They can be fitted by two xstar absorbers, with ionization $\log \left(\xi_{1,2}^{\prime} / \mathrm{erg} \mathrm{cm} \mathrm{s}^{-1}\right)=3.72$, 3.05 and velocity $v_{1,2}=0.267,0.225 \mathrm{c}$, confirming the presence of the UFO found by Parker et al. (2017b). The inclusion of these absorption features does not have any significant impact on the relativistic blurring parameters, indicating that the measurements made using relativistic reflection are robust.

(vi) The UFO absorption lines are prominent at low flux levels (MF and LF), which may result from the increasing ionization of the gas by the increasing X-ray flux.

(vii) A high-density disc model with number density $n_{\mathrm{e}}>10^{18.7} \mathrm{~cm}^{-3}$ can potentially fit the soft excess and lessen the supersolar iron abundance requirement for the reflection components.

\section{ACKNOWLEDGEMENTS}

J.J. acknowledges support by the Cambridge Trust and the Chinese Scholarship Council Joint Scholarship Programme (201604100032). A.C.F., M.L.P., and C.P. acknowledge support by the ERC Advanced Grant 340442. B.D.M. acknowledges support by the Polish National Science Center grant Polonez 2016/21/P/ST9/04025. W.N.A. acknowledges support from the European Union Seventh Framework Program (FP7/2013-2017) under grant agreement no. 312789, StrongGravity. G.M. acknowledges support from the European Union Seventh Framework Program (FP7/2007-2013) under grant agreement no. 312789, StrongGravity and the Spanish grant ESP2015-65597-C4-1-R. D.J.K.B. is supported by the Science and Technology Facilities Council (STFC). J.A.G. acknowledges the support from the Alexander von Humboldt Foundation. D.R.W. is supported by NASA through Einstein Postdoctoral Fellowship grant number PF6-170160, awarded by the Chandra X-ray Center, operated by the Smithsonian Astrophysical Observatory for NASA under contract NAS8-03060. D.J.W. acknowledges support from an STFC Ernest Rutherford fellowship. Based on observations obtained with XMM-Newton, an ESA science mission with instruments and contributions directly funded by ESA Member States and NASA. This project has made use of the Science Analysis Software (SAS), an extensive suite to process the data collected by the XMM-Newton observatory. This work made use of data from the NUSTAR mission, a project led by the California Institute of Technology, managed by the Jet Propulsion Laboratory, and funded by NASA. This research has made use of the NuSTAR Data Analysis Software (NuSTARDAS) jointly developed by the ASI Science Data Center and the California Institute of Technology.

\section{REFERENCES}

Allen D. A., Norris R. P., Meadows V. S., Roche P. F., 1991, MNRAS, 248, 528

Arnaud K. A., 1996, in Jacoby G. H., Barnes J., eds, ASP Conf. Ser. Vol. 101, Astronomical Data Analysis Software and Systems V. Astron. Soc. Pac., San Francisco, p. 17

Bachetti M. et al., 2014, Nature, 514, 202

Balucinska-Church M., McCammon D., 1992, ApJ, 400, 699

Boller T., Brandt W. N., Fabian A. C., Fink H. H., 1997, MNRAS, 289, 393

Boller T. et al., 2002, MNRAS, 329, L1

Boller T., Tanaka Y., Fabian A., Brandt W. N., Gallo L., Anabuki N., Haba Y., Vaughan S., 2003, MNRAS, 343, L89

Brenneman L. W. et al., 2011, ApJ, 736, 103

Brightman M. et al., 2013, MNRAS, 433, 2485

Buisson D. J. K. et al., 2018, MNRAS, 475, 2306

Cash W., 1979, ApJ, 228, 939

Chainakun P., Young A. J., Kara E., 2016, MNRAS, 460, 3076

Chartas G., Krawczynski H., Zalesky L., Kochanek C. S., Dai X., Morgan C. W., Mosquera A., 2017, ApJ, 837, 26 
Chiang C.-Y., Walton D. J., Fabian A. C., Wilkins D. R., Gallo L. C., 2015, MNRAS, 446, 759

Cunningham C., 1976, ApJ, 208, 534

Dauser T. et al., 2012, MNRAS, 422, 1914

Dauser T., Garcia J., Wilms J., Böck M., Brenneman L. W., Falanga M., Fukumura K., Reynolds C. S., 2013, MNRAS, 430, 1694

De Marco B., Ponti G., 2016, ApJ, 826, 70

De Marco B., Ponti G., Cappi M., Dadina M., Uttley P., Cackett E. M., Fabian A. C., Miniutti G., 2013, MNRAS, 431, 2441

De Marco B., Ponti G., Muñoz-Darias T., Nandra K., 2015, ApJ, 814, 50

De Marco B. et al., 2017, MNRAS, 471, 1475

Done C., Jin C., 2016, MNRAS, 460, 1716

Elvis M., 2000, ApJ, 545, 63

Fabian A. C., Vaughan S., 2003, MNRAS, 340, L28

Fabian A. C., Rees M. J., Stella L., White N. E., 1989, MNRAS, 238, 729

Fabian A. C., Miniutti G., Gallo L., Boller T., Tanaka Y., Vaughan S., Ross R. R., 2004, MNRAS, 353, 1071

Fabian A. C. et al., 2009, Nature, 459, 540

Fabian A. C. et al., 2013, MNRAS, 429, 2917

Feain I. J. et al., 2009, ApJ, 707, 114

Fürst F. et al., 2016, ApJ, 831, L14

Gallo L. C., Fabian A. C., 2011, MNRAS, 418, L59

Gallo L. C., Fabian A. C., 2013, MNRAS, 434, L66

Gallo L. C., Boller T., Tanaka Y., Fabian A. C., Brandt W. N., Welsh W. F., Anabuki N., Haba Y., 2004, MNRAS, 347, 269

Gallo L. C. et al., 2013, MNRAS, 428, 1191

García J., Kallman T. R., 2010, ApJ, 718, 695

García J. A., Fabian A. C., Kallman T. R., Dauser T., Parker M. L., McClintock J. E., Steiner J. F., Wilms J., 2016, MNRAS, 462, 751

George I. M., Fabian A. C., 1991, MNRAS, 249, 352

Haardt F., Maraschi L., 1993, ApJ, 413, 507

Hagino K., Odaka H., Done C., Tomaru R., Watanabe S., Takahashi T., 2016, MNRAS, 461, 3954

Harrison F. A. et al., 2013, ApJ, 770, 103

Israel G. L. et al., 2017a, Science, 355, 817

Israel G. L. et al., 2017b, MNRAS, 466, L48

Jansen F. et al., 2001, A\&A, 365, L1

Kaastra J. S., Bleeker J. A. M., 2016, A\&A, 587, A151

Kalberla P. M. W., Burton W. B., Hartmann D., Arnal E. M., Bajaja E., Morras R., Pöppel W. G. L., 2005, A\&A, 440, 775

Kallman T., Bautista M., 2001, ApJS, 133, 221

Kara E., Fabian A. C., Cackett E. M., Steiner J. F., Uttley P., Wilkins D. R., Zoghbi A., 2013a, MNRAS, 428, 2795

Kara E., Fabian A. C., Cackett E. M., Miniutti G., Uttley P., 2013b, MNRAS, 430, 1408

Kara E., Alston W. N., Fabian A. C., Cackett E. M., Uttley P., Reynolds C. S., Zoghbi A., 2016, MNRAS, 462, 511

Kosec P., Pinto C., Fabian A. C., Walton D. J., 2018, MNRAS, 473, 5680

Laor A., 1991, ApJ, 376, 90

Larsson J., Miniutti G., Fabian A. C., Miller J. M., Reynolds C. S., Ponti G., 2008, MNRAS, 384, 1316

Leighly K. M., 2001, in Peterson B. M., Pogge R. W., Polidan R. S., eds, ASP Conf. Ser. Vol. 224, Probing the Physics of Active Galactic Nuclei. Astron. Soc. Pac., San Francisco, p. 293

Leighly K. M., Moore J. R., 2004, ApJ, 611, 107

Leighly K. M., Mushotzky R. F., Nandra K., Forster K., 1997, ApJ, 489, L25

Marinucci A. et al., 2014a, MNRAS, 440, 2347

Marinucci A. et al., 2014b, ApJ, 787, 83

Martocchia A., Matt G., 1996, MNRAS, 282, L53

Martocchia A., Karas V., Matt G., 2000, MNRAS, 312, 817

Mas-Hesse J. M., Rodriguez-Pascual P. M., de Cordoba L. S. F., Boller T., 1994, A\&A, 283, L9

Matzeu G. A., Reeves J. N., Nardini E., Braito V., Costa M. T., Tombesi F., Gofford J., 2016, MNRAS, 458, 1311
Matzeu G. A., Reeves J. N., Braito V., Nardini E., McLaughlin D. E., Lobban A. P., Tombesi F., Costa M. T., 2017, MNRAS, 472, L15

Merloni A., Fabian A. C., 2001, MNRAS, 321, 549

Miniutti G., Fabian A. C., Goyder R., Lasenby A. N., 2003, MNRAS, 344, L22

Miniutti G., Panessa F., de Rosa A., Fabian A. C., Malizia A., Molina M., Miller J. M., Vaughan S., 2009, MNRAS, 398, 255

Morgan C. W., Kochanek C. S., Dai X., Morgan N. D., Falco E. E., 2008, ApJ, 689, 755

Murray N., Chiang J., Grossman S. A., Voit G. M., 1995, ApJ, 451, 498

Parker M. L. et al., 2014, MNRAS, 443, 1723

Parker M. L. et al., 2017a, MNRAS, 469, 1553

Parker M. L. et al., 2017b, Nature, 543, 83

Pinto C., Middleton M. J., Fabian A. C., 2016, Nature, 533, 64

Pinto C., Fabian A., Middleton M., Walton D., 2017, Astron. Nachr., 338, 234

Pinto C. et al., 2018, MNRAS, 476, 1020

Ponti G. et al., 2010, MNRAS, 406, 2591

Reis R. C., Miller J. M., 2013, ApJ, 769, L7

Reis R. C. et al., 2012, ApJ, 745, 93

Reis R. C., Miller J. M., Reynolds M. T., Fabian A. C., Walton D. J., Cackett E., Steiner J. F., 2013, ApJ, 763, 48

Reynolds C. S., Nowak M. A., 2003, Phys. Rep., 377, 389

Risaliti G. et al., 2013, Nature, 494, 449

Rodriguez-Pascual P. M., Mas-Hesse J. M., Santos-Lleo M., 1997, A\&A, 327,72

Ross R. R., Fabian A. C., 2005, MNRAS, 358, 211

Rossi S., Homan J., Miller J. M., Belloni T., 2005, MNRAS, 360, 763

Sani E., Lutz D., Risaliti G., Netzer H., Gallo L. C., Trakhtenbrot B., Sturm E., Boller T., 2010, MNRAS, 403, 1246

Svensson R., Zdziarski A. A., 1994, ApJ, 436, 599

Tan Y., Wang J. X., Shu X. W., Zhou Y., 2012, ApJ, 747, L11

Tanaka Y. et al., 1995, Nature, 375, 659

Tombesi F., Cappi M., Reeves J. N., Palumbo G. G. C., Yaqoob T., Braito V., Dadina M., 2010, A\&A, 521, A57

Tomsick J. A. et al., 2018, ApJ, 855, 3

Vasudevan R. V., Fabian A. C., 2007, MNRAS, 381, 1235

Vaughan S., Fabian A. C., 2004, MNRAS, 348, 1415

Walton D. J., Nardini E., Fabian A. C., Gallo L. C., Reis R. C., 2013, MNRAS, 428, 2901

Walton D. J. et al., 2014, ApJ, 788, 76

Walton D. J. et al., 2016, ApJ, 826, L26

Wang H., Zhou H., Yuan W., Wang T., 2012, ApJ, 751, L23

Wilkins D. R., Fabian A. C., 2012, MNRAS, 424, 1284

Wilkins D. R., Gallo L. C., 2015, MNRAS, 448, 703

Wilkins D. R., Gallo L. C., Grupe D., Bonson K., Komossa S., Fabian A. C., 2015, MNRAS, 454, 4440

Willingale R., Starling R. L. C., Beardmore A. P., Tanvir N. R., O’Brien P. T., 2013, MNRAS, 431, 394

Wilms J., Allen A., McCray R., 2000, ApJ, 542, 914

Wilms J., Reynolds C. S., Begelman M. C., Reeves J., Molendi S., Staubert R., Kendziorra E., 2001, MNRAS, 328, L27

Yan M., Sadeghpour H. R., Dalgarno A., 2001, ApJ, 559, 1194

Young A. J., Crawford C. S., Fabian A. C., Brandt W. N., O’Brien P. T., 1999, MNRAS, 304, L46

Zdziarski A. A., Johnson W. N., Magdziarz P., 1996, MNRAS, 283, 193

Zhou X.-L., Wang J.-M., 2005, ApJ, 618, L83

Zoghbi A., Fabian A. C., Uttley P., Miniutti G., Gallo L. C., Reynolds C. S., Miller J. M., Ponti G., 2010, MNRAS, 401, 2419

Zoghbi A., Uttley P., Fabian A. C., 2011, MNRAS, 412, 59

Zoghbi A., Fabian A. C., Reynolds C. S., Cackett E. M., 2012, MNRAS, 422,129

This paper has been typeset from a $\mathrm{T}_{\mathrm{E}} \mathrm{X} / \mathrm{L} \mathrm{T}_{\mathrm{E}} \mathrm{X}$ file prepared by the author. 